\title{
A stereoscopic PIV study on the behavior of near-field wingtip vortex structures
}

by

\author{
Hirofumi Igarashi
}

A thesis submitted to the graduate faculty in partial fulfillment of the requirements for the degree of

MASTER OF SCIENCE

\author{
Major: Aerospace Engineering \\ Program of Study Committee: \\ Hui Hu, Major Professor \\ Tom I-P. Shih \\ Chris R. Rehmann
}

\author{
Iowa State University \\ Ames, Iowa \\ 2011
}

Copyright (c) Hirofumi Igarashi, 2011. All rights reserved. 


\section{TABLE OF CONTENTS}

LIST OF TABLES ................... i iv

LIST OF FIGURES .....................

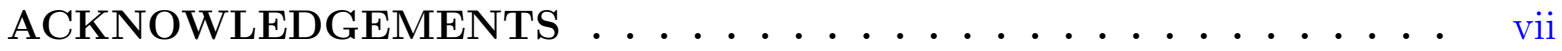

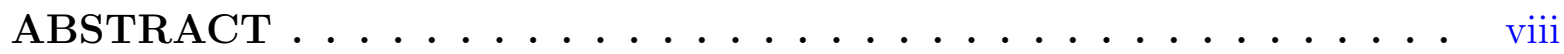

CHAPTER 1. INTRODUCTION ................ 1

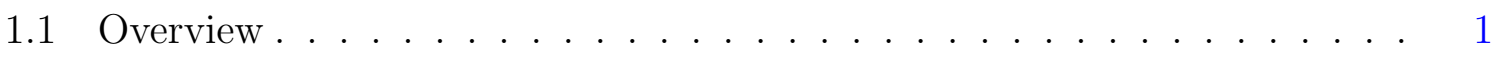

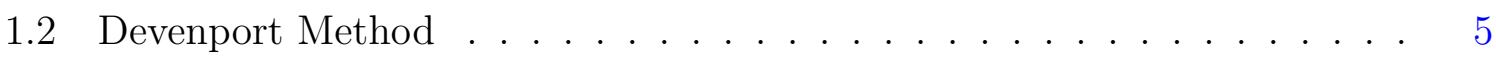

1.3 Vortex Center determination . . . . . . . . . . . . . . . . . 6

1.3.1 Vorticity Approach . . . . . . . . . . . . . . . . 7

1.3.2 Velocity Approach . . . . . . . . . . . . . . . . . . 9

1.4 SPIV Method . . . . . . . . . . . . . . . . . . 9

1.5 Data Reduction . . . . . . . . . . . . . . . . . . 10

CHAPTER 2. STEREOSCOPIC PIV AND SETUP . . . . . . . 12

2.1 Stereoscopic PIV System Introduction _ . . . . . . . . . . . . . . . . 12

2.1.1 Translation Method . . . . . . . . . . . . . 13

2.1.2 Angular-Displacement Method . . . . . . . . . . . . . 14

2.1.3 Angular-Displacement with Scheimpflug Condition . . . . . . . 16

2.1.4 Lens Distortion Compensation . . . . . . . . . . . . . . 17

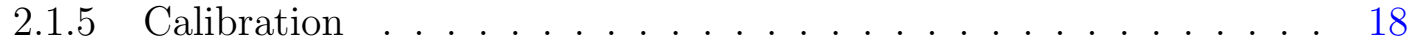

2.2 Error Analysis . . . . . . . . . . . . . . . . . . . . . . 19 
2.2.1 General Stereo Error analysis procedure . . . . . . . . . . . . 19

2.2.2 Geometric Error Analysis . . . . . . . . . . . . . . . . . . . 20

2.2 .3 Particle size . . . . . . . . . . . . . . . . 22

2.3 Experiment Setup . . . . . . . . . . . . . . . . . . . 23

2.3.1 Wind Tunnel and Stereoscopic PIV Set up . . . . . . . . . . 23

2.3.2 Illumination and Hardware . . . . . . . . . . . . . . . . . 25

CHAPTER 3. RESULTS . . . . . . . . . . . . . . 27

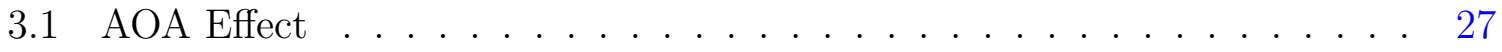

3.2 Downstream Effect . . . . . . . . . . . . . . . . . . 37

3.3 Devenport Method ...................... . . 44

3.4 Wall Effect . . . . . . . . . . . . . . . . . . . 46

CHAPTER 4. CONCLUSION . . . . . . . . . . . . . 52

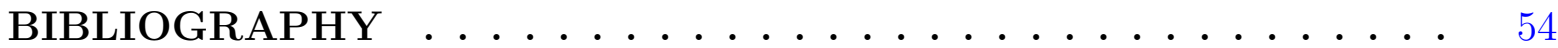




\section{LIST OF TABLES}

Table 3.1 Wandering amplitude data for SPIV method and Devenport method 45

Table $3.2 \quad$ Numerical data for SPIV method . . . . . . . . . . . . . 51 


\section{LIST OF FIGURES}

Figure $1.1 \quad$ Vorticity contour plot with linear and 2nd-order polynomial fit. . 8

Figure 1.2 Six nodal points for a 2nd order 2 dimensional polynomial fit. . . 8

Figure 1.3 Re-centered Velocity field is extracted from the original Velocity field. . . . . . . . . . . . . . . . . . . 10

Figure 2.1 Configuration for a translation method . . . . . . . . . . . . 13

Figure 2.2 Configuration for a angular-displacement method . . . . . . . . . 15

Figure 2.3 Configuration for a angular-displacement method with Scheimpflug Condition ...................... . . 16

Figure 2.4 Error ratio versus camera angle for angular-displacement method 22

Figure 2.5 Low speed wind tunnel used in the experiment. . . . . . . . . . . 24

Figure 2.6 The wing mount and laser position (optics not shown). . . . . . 25

Figure 2.7 Cross sectional view of wind tunnel test section. . . . . . . . . . 26

Figure $3.1 \quad$ AOA effect. . . . . . . . . . . . . . . . . . . . . . . . . 29

Figure 3.2 Normalized T.K.E. distributions for lower angle of attack. . . . . 33

Figure $3.3 \quad$ Normalized T.K.E. distributions for higher angle of attack. . . . 34

Figure 3.4 Normalized Reynolds stress distributions for lower angle of attack. 35

Figure 3.5 Normalized Reynolds stress distributions for higher angle of attack. 36

Figure $3.6 \quad$ Downstream effect. . . . . . . . . . . . . . . . . . . . . . . 39

Figure 3.7 Normalized T.K.E. distributions from 1.0 to 4.0 chord length

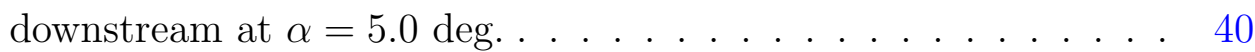


Figure 3.8 Normalized T.K.E. distributions for 1.0 and 2.0 chord length

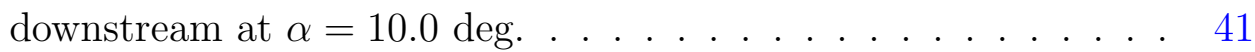

Figure 3.9 Normalized Reynolds Stress distributions from 1.0 to 4.0 chord length downstream at $\alpha=5.0$ deg. . . . . . . . . . . . . . . 42

Figure 3.10 Normalized Reynolds Stress distributions from 1.0 to 4.0 chord length downstream at $\alpha=10.0$ deg. . . . . . . . . . . . . 43

Figure 3.11 Re-centered tangential velocity profiles by SPIV and Devenport methods. . . . . . . . . . . . . . . . 45

Figure 3.12 Effect of upwash by the additional walls. . . . . . . . . . . . . . 46

Figure 3.13 Correlation of wall-to-tip distance and vortex upward travel. . . 47

Figure 3.14 T.K.E. distributions for wall effect. . . . . . . . . . . . . . . . . 49

Figure 3.15 Normalized Reynolds Stress distributions for wall effect. . . . . . 50 


\section{ACKNOWLEDGEMENTS}

I would like to express my thanks to everyone who helped me with various aspects of conducting research and the writing of this thesis. My major professor Dr. Hui Hu for all his guidance and support, my committee members Dr. Tom Shih and Dr. Chris

Rehmann, Dr. Hu's research group members, and Mr. Bill Rickard. I would additionally like to thank my family and fiancée for all their love and support through all the many years of my education. 
viii

\section{ABSTRACT}

Wingtip vortex flow is of great importance because of its effect on practical problems such as landing separation distances for aircraft, blade/vortex interactions on helicopter blades, and propeller cavitations on ships. Extensive investigations have been conducted to improve the understanding of the tip vortex structure and its dissipation or persistence

analytically, numerically, and experimentally. The universal feature of the water/wind tunnel generated wing tip vortex reported in the past is vortex wandering - the slow side-to-side movement of the wing-tip vortex core behind the wing. Thus, a primary result of wandering is that fixed probe measurements of velocity and pressure cannot be trusted at distances more than one chord downstream of the wing.

For reliable data, the current study investigates the behavior and structure of the near-field wing-tip vortex generated by a square-tipped, rectangular NACA0012 wing by using the stereoscopic Particle Image Velocimetry (SPIV) technique. SPIV is a spatially resolved, instantaneous, three velocity component non-intrusive measurement technique used to conserve the three key feature of the wing-tip vortex during the measurement - small vortex core dimension, core structure, and strong unsteadiness of the core flow, which wasn't possible with classical instrumentations.

One of the great advantages of SPIV over the classical technique is that the vortex wandering can be removed by tracking the center of the vortex in every SPIV frame. By tracking the center of the vortex, the wandering and turbulence in the vortex can be separated. The results show that after re-centering the velocity field, the T.K.E. and Reynolds stress distributions become lower by more than twice at 4.0c downstream. 
This suggests that the vortex itself is laminar after the rollup and the higher turbulence intensity in the vortex core, reported in past studies, is mainly due to vortex wandering. This SPIV method is applied to investigate the angle of attack effect, downstream effect, and wind tunnel wall effect. Past studies suggest that the vortex rollup is completed about two chord lengths behind the wing trailing edge. The SPIV method confirmed that the vortex rollup is completed at 3.0c downstream for $\alpha=5.0^{\circ}$ and $4.0 \mathrm{c}$ for $\alpha=10.0^{\circ}$ by observing the re-centered Reynolds stress distributions. As for correcting the velocity profile, Devenport et al. (1996) found an analytical way to predict the wandering free velocity profile using the Reynolds stress at the vortex center. The velocity profile predicted by the Devenport et al. (1996) method is compared with the SPIV re-centered velocity profile. The results show that the two profiles agree with each other very well in the vicinity of core when the vortex wandering is large enough (about $20 \%$ of vortex core radius). 


\section{CHAPTER 1. INTRODUCTION}

\subsection{Overview}

The behavior of strong, coherent wingtip vortex structures is of great importance to various commercial and military applications. One well-known problem is the hazardous effect of trailing wingtip vortices on flight safety and airport capacity. From a military standpoint, there are many issues associated with the effects of wingtip vortex structures on dynamics of towed vehicles, tail buffeting, and icing arrays. Blade/vortex interaction on helicopter blades can impact performance and cause undesirable noise and vibration. The study of wingtip vortices is not only of great engineering importance but also of great scientific interest. It continues to be a perplexing problem for computational scientists because of the presence of large gradients of velocity and pressure in all three dimensions, especially in the near field, at high Reynolds numbers.

A good physical understanding is essential in order to guide a variety of applications. Thus, a detailed knowledge of the transient behavior of wing-tip vortex dynamics in the near field is highly desired. Numerous experimental, theoretical and computational investigations have been conducted in recent years to improve the basic understanding of wing-tip vortex structures, as well as its control. Devenport et al. (1996) investigated vortex structure in the range of $\mathrm{X} / \mathrm{C}=4$ to 29 downstream of a NACA 0012 airfoil with a blunt tip, at $\operatorname{Re}=5.35 \times 10^{5}$. These authors used a miniature, four-sensor hot-wire probe, and showed a deficit profile of approximately $84 \%$ of the free-stream velocity. They reported that the flow outside the vortex core was dominated by the remainder 
of the wing wake, which wound into an ever increasing spiral, and the turbulence stress levels varied along the wake spiral in response to the varying rate of strain imposed by the vortex.

Chow et al. (1997) investigated the wing-tip vortex flow of a NACA 0012 airfoil model with a rounded tip at $\operatorname{Re}=4.6 \times 10^{6}$ and $\alpha=10^{\circ}$, making measurements with a seven-hole pressure probe and with a triple-hot-wire probe. They indicated a high level of axial velocity, in excess of $1.7 U_{\infty}$, at all measurement locations. They also reported that the turbulence intensity in the vortex can be as high as $24 \%$, but it decayed quickly with streamwise distance because of the stabilizing effect of the nearly solid-body rotation in the vortex-core. Ramaprian and Zheng (1993) observed no axial velocity excess for a tip vortex generated by a rectangular, square-tipped NACA 0015 wing with $\operatorname{Re}=1.8 \times 10^{5}$ at $\alpha=10^{\circ}$. They used a three-component laser Doppler anemometer. The inner part of the vortex was, however, found to be nearly axisymmetric within $\mathrm{X} / \mathrm{C}=2.0$ and exhibited a universal structure of asymptotic trailing vortices.

More recently, Birch et al. (2004) examined the flow structure both along the tip and in the near field (up to $\mathrm{X} / \mathrm{C}=2.5$ ) behind a square-tipped, rectangular NACA 0015 wing at $\mathrm{Re}=2.1 \times 10^{5}$ for angles of attack ranging from 2 to 19 degrees. Their measurements were produced from a miniature, seven-hole pressure probe and a triple-hot-wire probe. The circulation was observed to have a local maximum at $\mathrm{X} / \mathrm{C}=0.05$ and remained virtually unchanged up to $\mathrm{X} / \mathrm{C}=2.5$. The vortex flow was self-similar and axisymmetric for $\mathrm{X} / \mathrm{C}=0.5$. The lift-induced drag was also computed and compared with the windtunnel force-balance Vortex wandering, the slow side-to-side movement of the wing-tip vortex core, which has been found to be a universal feature of wind tunnel generated wing-tip vortex structures.

Jaquin et al. (2001) proposed four possible causes for vortex wandering: the vortex could be un-stabilized by wind-tunnel free-stream unsteadiness, turbulence in the surrounding shear layer, co-operative instabilities, or propagation of unsteadiness from the 
model. In any case, its most significant effect is to obscure point-wise measurements by smearing the vortex core. Corsiglia et al. (1973) reported that vortex wandering could be overwhelming, with the wandering amplitude being several times of vortex core diameter. Devenport et al. (1996) developed an analytical technique to correct meanvelocity profiles measured with a fixed hot-wire probe, thereby providing quantitative estimates of wandering amplitude and its contributions to Reynolds stress. This process involves forming a "corrected" mean velocity field from the measured version using guessed levels of wandering amplitude. The corrected field is then artificially subjected to wandering, described by a bi-variate Gaussian probability density function (PDF) for vortex position, and the resulting Reynolds stresses at the vortex center are compared with the actual measured values. The wandering amplitudes are then adjusted iteratively until the calculated and measured values converge. Using their wandering correction theory, they found that the amplitude of wandering varied linearly between 0.1 and 0.4 vortex core radii at stream-wise distances of 5 to 36 chord lengths, and at its highest level, wandering was responsible for a $12 \%$ and $15 \%$ error in the measured core radii and peak tangential velocity respectively. They concluded that the vortex core in their experiments was laminar and that velocity fluctuations in the vortex core region were entirely due to wandering. Rokhsaz et al. (2000) investigated wandering of a tip-vortex from a rectangular flat plate airfoil in a water tunnel and showed that wandering increased with angle of attack and therefore vortex strength, the opposite to the finding of Devenport et al. (1996) who showed a $15 \%$ reduction in wandering amplitude with an increase in vortex strength of $85 \%$.

These studies have uncovered useful information, but some of the inconsistencies noted above raise questions. The majority of the previous studies used point-wise flow measurement techniques such as pressure probes, hot-wire anemometry, and laser Doppler anemometer. A common shortcoming of such point-wise measurements is the inability to provide spatial structure of the unsteady vortices. Full field measurements 
are needed to effectively reveal the transient behavior of the wing-tip vortex structures. Temporally-synchronized and spatially-resolved flow field measurements are highly desirable in order to elucidate underlying physics. Advanced flow diagnostic techniques such as high-resolution stereoscopic Particle Image Velocimetry (SPIV) used in the present study are capable of providing such information.

Based on the flow field measurements with a conventional two-dimensional PIV system, Heyes et al. (2004) evaluated wandering effects by re-centering PIV data. They found that the Devenport et al. (1996) assumption of using a bi-variate normal probability density function could be valid, and their corrections were in good agreement with those predicted by the Devenport et al. (1996) method. They found a $12.5 \%$ over prediction of the core radius and a $6 \%$ under-prediction of the peak tangential velocity. The errors were larger for lower angles of attack. They also found that the wandering amplitude increases linearly with streamwise distance; a linear reduction was found by increasing the angle of attack, so that they concluded that the mechanism responsible for wandering is not self-induced, as had been proposed by Rokhsaz et al. (2000), but rather that the vortex is responding to an external perturbation, such as the background turbulence level, to which the tip vortex becomes less susceptible as the vortex strength is increased. It should be noted the PIV measurement results reported in Heyes et al. (2004) were obtained by using a conventional 2-D PIV system. It is well known that a conventional 2-D PIV system is only capable of recording the projection of velocity into the plane of the laser sheet. That means the out-of-plane velocity component is lost while the in-plane components may be affected by an unrecoverable error due to the perspective transformation (Prasad and Adrian (1993)). For the highly three-dimensional flow fields like wingtip vortex, the two-dimensional measurement results may not be able to reveal their three-dimensional features successfully.

In the present study, a high-resolution Stereoscopic Particle Image Velocimetry (SPIV) system, which is capable of achieving quantitative measurements of all three components 
of instantaneous flow velocity vectors, is used to conduct detailed flow field measurements to quantify the transient behavior of the wing-tip vortex generated from a rectangular NACA0012 airfoil. Because the field is instantaneous, wandering does not smear the vortex. The trajectory of the wing-tip vortex structures in the cross planes at different downstream locations will be revealed directly as a time sequence of the instantaneous SPIV measurements. The underlying physics and characteristics of vortex wandering is revealed in great detail.

\subsection{Devenport Method}

Before the establishment of the modern flow diagnostic techniques like PIV and Stereoscopic PIV, only the single-point measurement probes were available. To recover the wandering free profile of the vortex, Devenport et al. (1996) devised the technique to estimate the wandering amplitude and reconstruct the vortex profile. This technique requires fitting of the time-averaged tangential velocity profile with the following series

$$
V_{\theta m}\left(0, z_{p}\right)=\sum_{i=1}^{n} \frac{D_{i}}{z_{p}}\left[1-\exp \left(\frac{-z_{p}^{2}}{c_{i}^{2}}\right)\right]
$$

where $D_{i}$ and $c_{i}$ are the coefficients to be determined by the curve fitting. By assuming that flow vicinity of the vortex core is axisymmetric and the vortex center is predicted by the bivariate probability density function of the form,

$$
p\left(y_{v}, z_{v}\right)=\frac{1}{2 \pi \sigma_{y} \sigma_{z}\left(1-\rho^{2}\right)^{1 / 2}} \exp \left[\frac{-1}{2\left(1-\rho^{2}\right)}\left(\frac{z_{v}^{2}}{\sigma_{z}^{2}}+\frac{y_{v}^{2}}{\sigma_{y}^{2}}-\frac{2 \rho y_{v} z_{v}}{\sigma_{y} \sigma_{z}}\right)\right]
$$

Devenport et al. (1996) suggests that the tangential velocity is accurately described by series

$$
V_{\theta}(y, z)=\sum_{i=1}^{n} \frac{A_{i} a_{i}^{2}}{2 r}\left[1-\exp \left(-\frac{y^{2}+z^{2}}{a_{i}^{2}}\right)\right]
$$


where $A_{i}$ and $a_{i}^{2}$ are derived from the functions of fitted coefficients $\left(D_{i}\right.$ and $\left.c_{i}\right)$. The wandering amplitudes $\left(\sigma_{y}\right.$ and $\sigma_{z}$ ) and correlation of those two variables $(\rho)$ are determined by iterative process of matching the calculated and measured apparent stresses $\overline{v_{m}^{2}}, \overline{w_{m}^{2}}$ and $\overline{v w_{m}}$ at the vortex center. Derived by Devenport et al. (1996), the calculated apparent Reynolds stresses at the mean vortex center are found by

$$
\overline{u v_{m}}(0,0)=\int_{-\infty}^{\infty} \int_{-\infty}^{\infty} p\left(y_{v}, z_{v}\right) U\left(-y_{v},-z_{v}\right) V\left(-y_{v},-z_{v}\right) d y_{v} d z_{v}-U_{m} V_{m}
$$

with like expressions for $\overline{u_{m}^{2}}, \overline{v_{m}^{2}}, \overline{w_{m}^{2}}, \overline{v w_{m}}$ and $\overline{u w_{m}}$. $\left(y_{v}, z_{v}\right)$ in the Eqn.1.4 are the current location of the vortex center. In general, calculated Reynolds stresses don't agree with measured Reynolds stresses with initial guessed wandering amplitudes. Therefore $\sigma_{y}, \sigma_{z}$ and $\rho$ are adjusted and the procedure repeated until they match. The wandering amplitudes are adjusted in the following way till the calculated stresses converge to the measured stresses.

$$
\begin{aligned}
& \sigma_{y_{\text {new }}}^{2}=\sigma_{y_{\text {old }}}^{2}{\overline{w_{m_{\text {meas }}}^{2}}}_{\bar{w}_{m_{\text {calc }}}^{2}} \\
& \sigma_{z_{\text {new }}}^{2}=\sigma_{z_{\text {old }}}^{2}{\overline{v_{m_{\text {meas }}}^{2}}}_{\bar{v}_{m_{\text {calc }}^{2}}}
\end{aligned}
$$

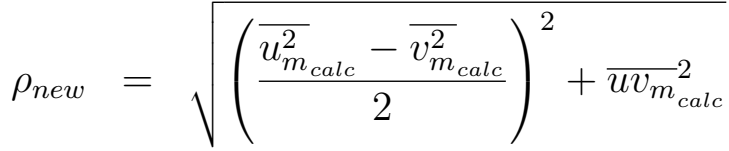

The results of Devenport et al. (1996) is compared with the results of SPIV method in Section 3.3.

\section{$1.3 \quad$ Vortex Center determination}

Upon the SPIV data reduction, there are two approaches to determine the vortex center. The first approach is to determine the maximum (positive or negative) vorticity, 
as the study done by Cerreteli and Williamson (2003). The other approach is to find the location where the cross stream velocities go to zero, i.e., $v=w=0$. The current study investigated both approaches but the zero cross velocity method is used for finding the center since it physically made more sense.

\subsubsection{Vorticity Approach}

As the study done by Cerreteli and Williamson (2003), the vortex center can be determined from the vorticity field as the peak (positive or negative) vorticity location. The current study, the vorticity $(\omega)$ calculation was obtained by use of the fourth order accurate finite difference method suggested by Cohn and Koochesfahani (2000) as follows.

$$
\omega=\frac{\partial v}{\partial x}-\frac{\partial u}{\partial y}
$$

where

$$
\begin{aligned}
& \left(\frac{\partial v}{\partial x}\right)_{i, j}=\frac{-v_{i+2, j}+8 v_{i+1, j}-8 v_{i-1, j}+v_{i-2, j}}{12 \Delta x} \\
& \left(\frac{\partial u}{\partial y}\right)_{i, j}=\frac{-u_{i, j+2}+8 u_{i, j+1}-8 u_{i, j-1}+u_{i, j-2}}{12 \Delta y}
\end{aligned}
$$

This method, as is, introduces an error in the center position because the vorticity value will naturally lie at discrete node location (Figure 1.1); therefore, sub-grid interpolation is desired. After the maximum negatively or positively vorticity node is determined, a second order two dimensional polynomial fit, as described in Equation 1.11, is performed with the neighboring 6 node values as shown in Figure 1.2.

$$
R(x, y)=A x^{2}+B y^{2}+C x y+D x+E y+F
$$

To determine the location of the extrema, the partial derivative with respect to $y$ and $z$ of Eqn 1.11 is taken and set to zero for solving $\mathrm{x}$ and $\mathrm{y}$ as shown below. 


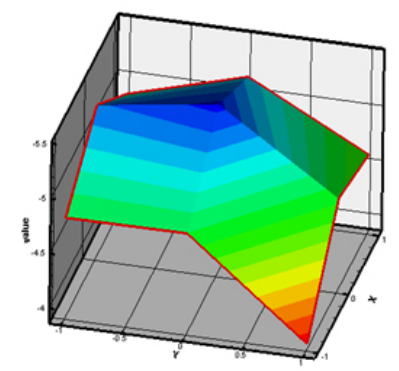

(a) Linear fit.

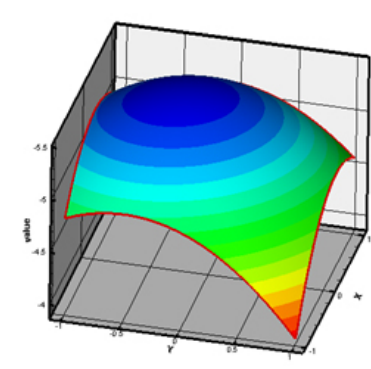

(b) 2nd-order polynomial fit.

Figure 1.1: Vorticity contour plot with linear and 2nd-order polynomial fit.

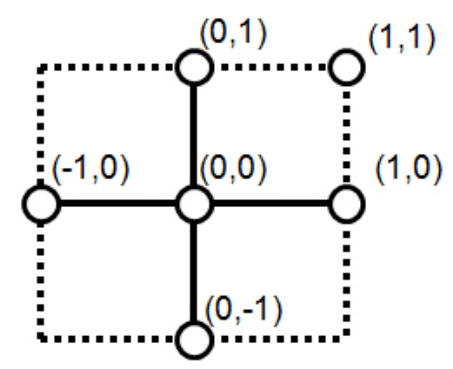

Figure 1.2: Six nodal points for a 2nd order 2 dimensional polynomial fit.

$$
\begin{aligned}
& \frac{\partial R}{\partial x}=2 A x+C y+D=0 \\
& \frac{\partial R}{\partial y}=2 B y+C x+E=0
\end{aligned}
$$

For the vortex wandering case, the cross flow velocity at the peak vorticity is non-zero since the vortex is wandering at certain velocity. To enforce the $v=w=0$ condition, the non zero-velocity at the peak is subtracted from the rest of the velocity field. Since the vorticity is linear, the vorticity would not change before/after subtracting the velocity. 


\subsubsection{Velocity Approach}

First, the vortex center is estimated by finding the maximum negative vorticity around the middle of the measured plane. Then, the first $7.0 \times 7.0 \mathrm{~mm}$ grid with $8 \times 8$ nodes are formed with maximum vorticity in the center and the cross stream velocities are interpolated to the minimum values. After determining the minimum cross stream velocity with the $7.0 \times 7.0 \mathrm{~mm}$ grid, the new grid is formed twice to a $5.0 \times 5.0 \mathrm{~mm}$ grid with $51 \times 51$ nodes and further down to a $1.0 \times 1.0 \mathrm{~mm}$ grid with $101 \times 101$ nodes. As the program generates new grids, the grid size gets smaller and the grid gets finer. The velocities on the new grid nodes are linearly interpolated from nearby node values in the old grid since the velocity profile is close to linear in the vortex core.

\subsection{SPIV Method}

In this section, the method used to extract the wandering free vortex velocity field is described; hereafter, referred to as the "SPIV method" to distinguish from the Devenport et al. (1996) correction method. SPIV data presented in this study is the result of 498 instantaneous SPIV frames, taken at the rate of 4 frames per second. Due to the camera RAM capacities, only a batch of 83 frames is recorded at one time. To make up a total of 498 frames, the experiments were immediately repeated five more times. Since the data acquisition rate was far below the frequency of the vortex motion, the velocity fields shown in this study is the time average of 498 instantaneous frames, or about 120 seconds.

To extract the wandering free vortex velocity field, it is necessary to keep track of the vortex center at every instantaneous SPIV frame. First, the center of the vortex is determined by the velocity approach method described in the section 1.3.2. Then a 50 to $40 \mathrm{~mm}$ square velocity field, depending on the case, is extracted with the vortex center at $(0,0)$ as shown in Figure 1.3. By repeating this method for all the frames, these square 


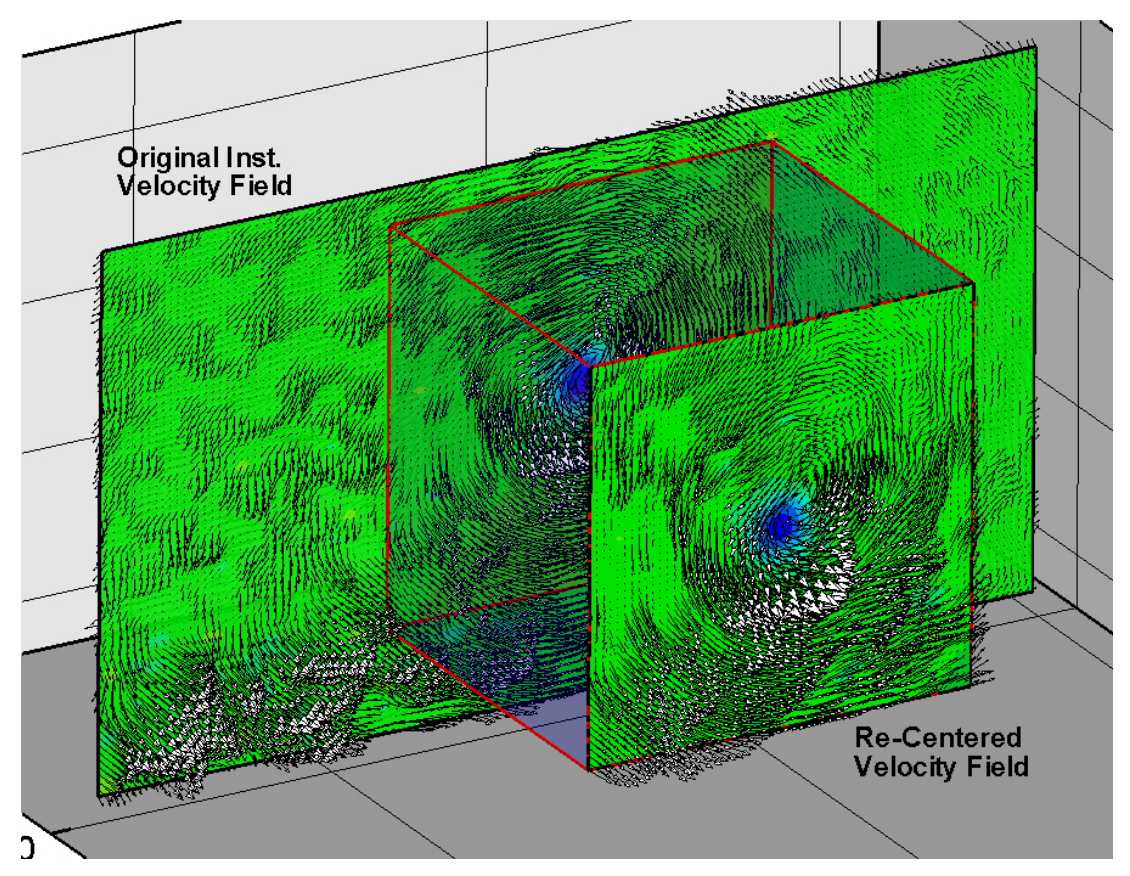

Figure 1.3: Re-centered Velocity field is extracted from the original Velocity field.

velocity fields can be considered as the instantaneous "re-centered" velocity fields.

\subsection{Data Reduction}

For both non re-centered and re-centered SPIV results, the turbulent kinetic energy, T.K.E., and Reynolds stresses are calculated as follow;

T.K.E.:

$$
T . K . E .=\frac{1}{2} \rho\left({\overline{u^{\prime}}}^{2}+{\overline{v^{\prime}}}^{2}+{\overline{w^{\prime}}}^{2}\right)
$$

where

$$
\begin{aligned}
\overline{u^{\prime}} & =\sqrt{\sum_{i=1}^{N}\left(u_{i}-U\right)^{2} / N} \\
U & =\sum_{i=1}^{N} u_{i} / N
\end{aligned}
$$

likewise for other velocities. 
Reynolds stress:

$$
\tau=-\rho \overline{u^{\prime} v^{\prime}}=-\rho \sum_{i=1}^{N} \frac{\left(u_{i}-U\right)\left(v_{i}-V\right)}{N}
$$

In order to complete the Devenport method, the measured Reynolds stresses at the vortex center is needed. In this study, the measured Reynolds stress is determined by using the Equation 1.17 for the non re-centered SPIV results. 


\section{CHAPTER 2. STEREOSCOPIC PIV AND SETUP}

Recent advances in PIV have been directed towards obtaining three-dimensional (3D) measurements of fluid velocity to allow the study of more complex fluid systems. Several methods which have been successfully demonstrated include holographic PIV (HPIV), dual-plane PIV and stereoscopic PIV. Of the three techniques, HPIV is the most complex and requires a significant investment in equipment and the development of advanced data processing techniques. Along with Dual-plane PIV, Stereoscopic PIV is also less complex than HPIV using only a single light sheet and two cameras, although the technique does use some image processing. The present study used the stereoscopic PIV to investigate a wing-tip vortex generated by the airfoil in a wind tunnel.

\subsection{Stereoscopic PIV System Introduction}

Classical particle image velocimetry (PIV) uses a single camera facing parallel to the illuminated plane. The resulting vector field suffers from two deficiencies (Prasad and Jensen (1995)). First, the out-of-plane velocity component is lost. The vectors are the two-dimensional projections on the object plane of the full three dimensional vectors. Second, the measured in-plane components are themselves contaminated by perspective error resulting from a local nonzero out-of-plane component. Stereoscopic imaging eliminates both of the above shortcomings. Simultaneous views from two different off-axes directions provide sufficient information to extract the out-of-plane component as well as to correct for the errors in the in-plane components (Prasad and Jensen (1995)). 
There are two basic configurations of stereoscopic PIV systems, translation method and angular-displacement method, and they are described in the following sections.

\subsubsection{Translation Method}

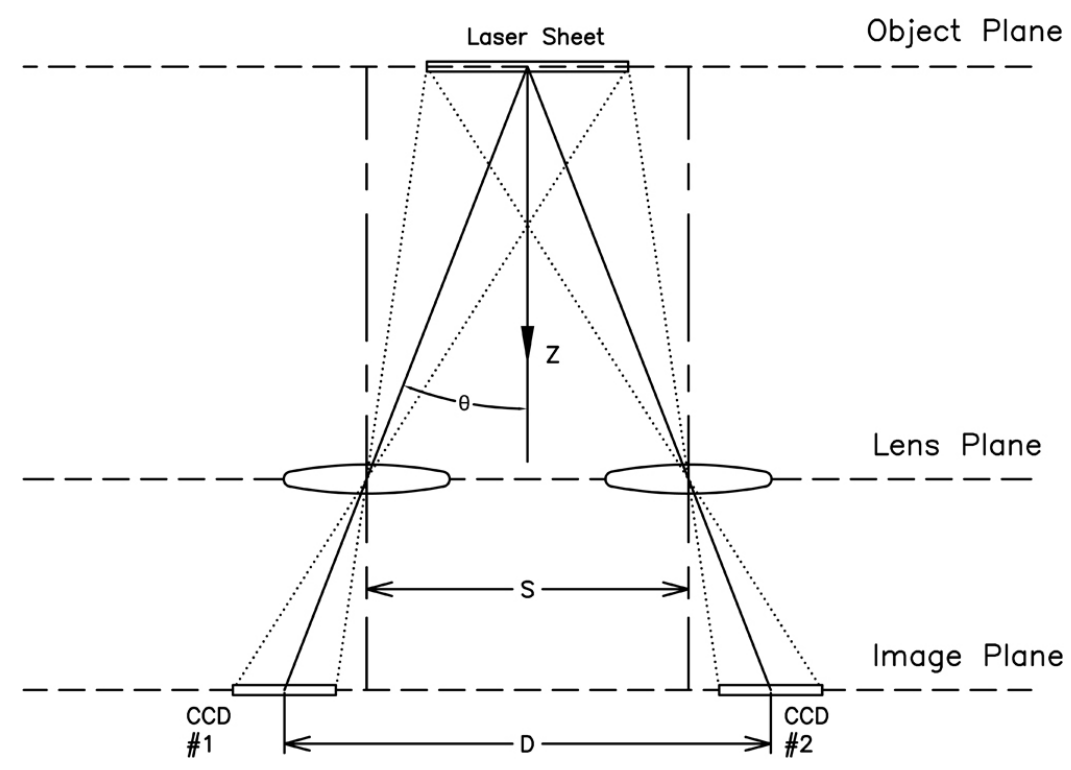

Figure 2.1: Configuration for a translation method

In the translation method, the axis of two cameras are placed parallel to each other and orthogonal to the object plane, or the light sheet as shown in Figure 2.1. The primary advantage of the translation method is its simplicity. Because the object plane, lens plane and image plane are all parallel to each other, the magnification is invariant at every location across both CCDs. Therefore the vector field resulting each view can be readily combined without any additional manipulation. Since the focal plane and laser sheet are also parallel, good image focus is achieved without reducing aperture diameter hence more scattering light will be reached on CCDs, reducing mean bias error in PIV interrogation (see section 2.2.3 for more). One of the disadvantages raised in the past was the small common area viewed by each camera; however, the common area can be 
maximized by moving each CCDs away from lens axis as shown in Figure 2.1 such that:

$$
D=(1+M) S
$$

where $\mathrm{D}$ is the distance between the centers of the CCDs, $\mathrm{S}$ is the distance between the camera lens axes and $\mathrm{M}$ is the camera magnification. A more serious difficulty with the translation system is that there is an upper bound to the off-axis angle $\theta$ subtended by the center of the region of interest to the center of the lens. This restriction arises purely from the design of the lens; if the lense are separated by a value that is too large for a

given $d_{0}$, the lens performance degrades as it is forced to operate at the outer limit of its specification. The lens performance can be seen on the modulation transfer function $(\mathrm{MTF})$. Since the relative error in the out-of-plane component decreases when the offaxis angle $\theta$ increases, the translation system is somewhat limited in the accuracy of the out-of-plane component. To increase the out-of-plane component accuracy without losing the lens quality, the angular method has been established and described in the following subsection.

\subsubsection{Angular-Displacement Method}

The restriction on the accuracy of the out-of-component imposed by the translation method is removed in an angular-displacement method as shown in Figure 2.2. For the angular-displacement method, the two camera axes are no longer parallel to each other but are rotated inward. Since a mean angle subtended by the object to the lens is zero, the largest angle subtended by the outermost edge of the object to the lens is well within lens specifications even for large $\theta$. Because the lenses are no longer operating at the extreme of their specification range, larger off axis angles are possible with the angulardisplacement system. A disadvantage is, unlike translation method, the object plane is no longer parallel to the lens plane. Therefore it becomes more difficult to obtain well focused image across the CCDs. One simple solution is to increase the $f$ number, or the 


\section{5}

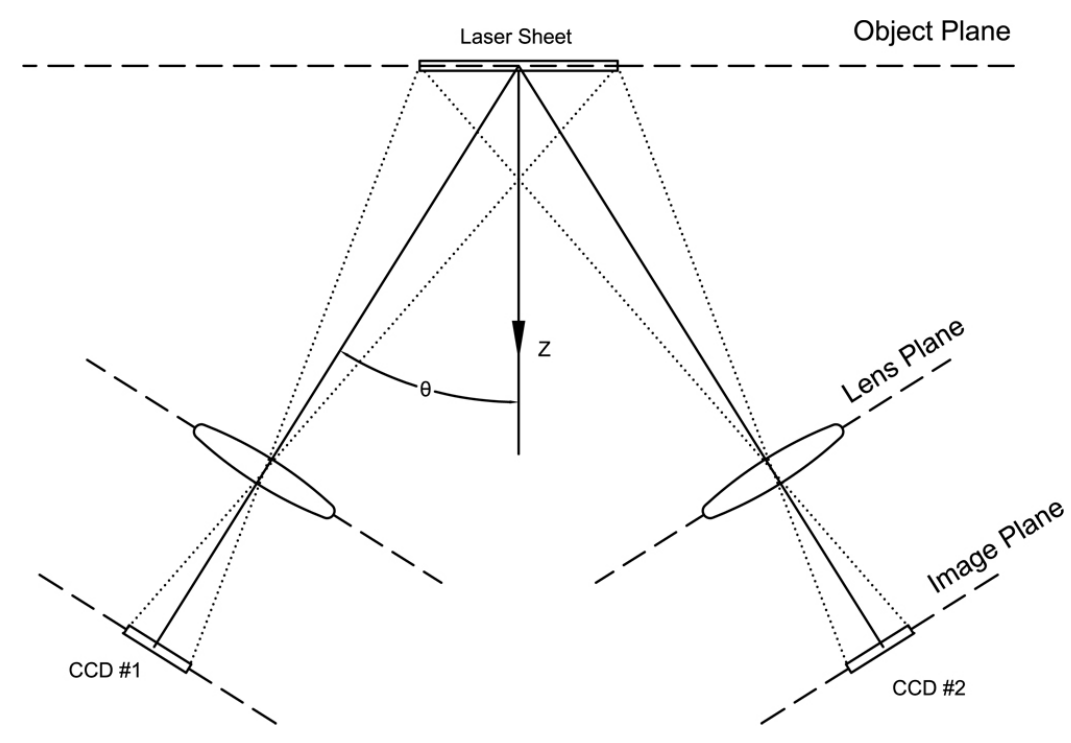

Figure 2.2: Configuration for a angular-displacement method

depth of a focal field. According to Adrian (1991), the depth of focal field $\delta z$ is given as:

$$
\delta z=4\left(1+M^{-1}\right)^{2} f \#^{2} \lambda
$$

where $\mathrm{M}$ is the camera magnification, $f \#$ is the $f$-number and $\lambda$ is the wavelength of the illuminating laser (532nm for the present study). According to Lawson and Wu (1997b), the optimum performance for the angular displacement method will be obtained by using camera angles $\theta$ of between $20^{\circ}$ and $30^{\circ}$ and camera $f$ numbers of $f 16$ and higher. The camera angle can be wider than the translation method so that the accuracy of the third component velocity vector is higher but still has the error ratio of about 2.0 (reference to the error vs angle). Moreover, the larger $f$ number, i.e., smaller aperture makes less scattered light to reach to CCDs. Hence the particle size cast on the CCD will be small which increases the mean bias error (Prasad et al. (1992)). To overcome this shortcoming of the angular-displacement method, the Scheimpflug condition is enforced to get a clear image on the image plane with a small $f$ number. 


\subsubsection{Angular-Displacement with Scheimpflug Condition}

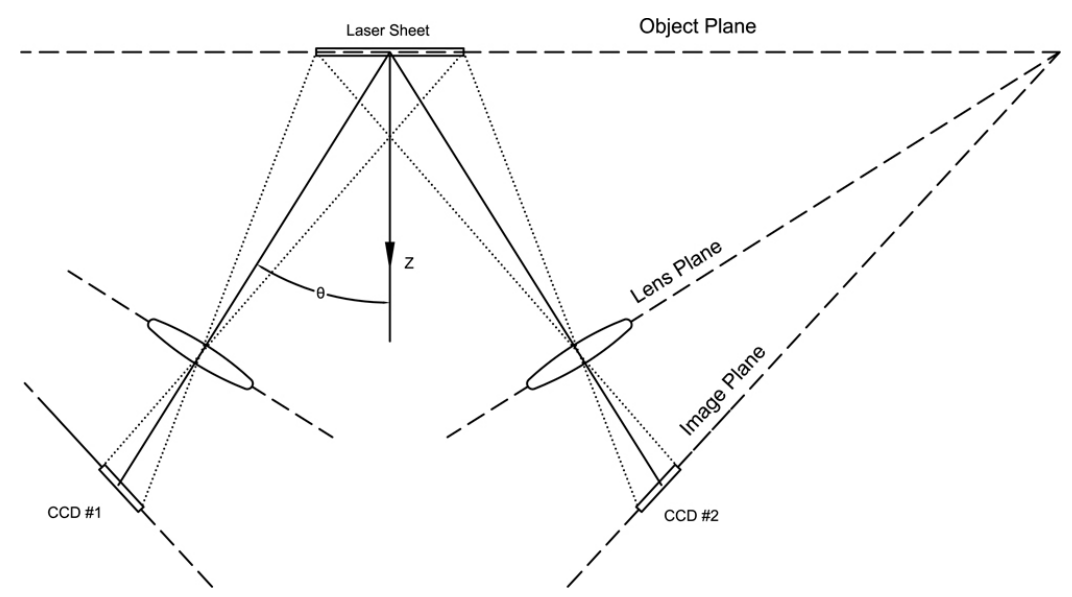

Figure 2.3: Configuration for a angular-displacement method with Scheimpflug Condition

The shortcoming of the angular-displacement is that it's difficult to obtain well focused image across the CCD since the object plane is no longer parallel to the lens plane. To overcome the shortcoming, the use of Scheimpflug condition was suggested by Hinsch (1995) and Prasad and Jensen (1995). First discovered by Scheimpflug (1904), the Scheimpflug condition is the condition such that the object plane, lens plane, and image plan is collinear, as shown in Figure 2.3. The derivation of Scheimpflug condition is provided by Prasad and Jensen (1995) as follow:

Placing the origin of the coordinate system at the center of the lens $\mathrm{O}$, we have the following expressions,

for the lens plane,

$$
z=0
$$

for the object plane,

$$
z=x \tan \theta-d_{o}
$$


for the image plane,

$$
z=-x \tan \alpha+d_{i}
$$

where $d_{o}$ and $d_{i}$ are the nominal object and image distances, respectively. (In the above expressions, $d_{o}, d_{i}, \theta$, and $\alpha$ are all positive quantities.) Any point lying in the object plane $\left(x_{o}, z_{o}\right)$ will form an image at the point $\left(x_{i}, z_{i}\right)$ as shown in Figure2.3, such that

$$
\frac{x_{o}}{z_{o}}=\frac{x_{i}}{z_{i}}
$$

Using Eqs. 2.2-2.4, we can readily show that

$$
\frac{1}{z_{i}}-\frac{1}{z_{o}}=\frac{1}{d_{o}}+\frac{1}{d_{i}}\left(=\frac{1}{f}\right)
$$

which is the condition that particle images will be in sharp focus in the image plane, $f$ being the focal length of the lens. Since the object plane and image plane are not parallel to each other, the magnification is significantly nonuniform even with the use of the Scheimpflug condition. To reduce the magnification in the image plane, the author may propose the use of a lens with longer focal length.

\subsubsection{Lens Distortion Compensation}

According to Soloff et al. (1997), accuracy of recording particle locations and their respective displacement depends on two types of optical aberrations: focusing aberrations (which cause the particle images to be enlarged, making it more difficult to measure displacements accurately) and image distortion (which creates a nonlinear relationship between the location of a particle and the location of its image). Focusing aberrations include spherical aberration, astigmatism and coma (Jenkins and White, 1957). Image distortion refers to any agency that causes the magnification to vary as a function of 
position in the field of the image. There are three agencies that cause the magnification in an experiment to carry. The first is design imperfection in the lens. The second is refraction caused by anything, including windows and fluid interfaces, that lies between the object plane in the illuminating light sheet and the image plane. The third is misalignment of the image recording plane with respect to the object plane (Soloff et al. (1997)). A conventional PIV system that uses a single camera oriented orthogonally to the interface records particle images that are, for the most part, free of radial distortions owing to the small off-axis angles subtended by the particles to the camera. To ensure that the two cameras continue to enjoy an orthogonal orientation with respect to the liquid-air interface in angular-displacement method, Prasad and Jensen (1995) redesigned the wall of the test section to incorporate a triangular prismatic section. Prasad and Jensen (1995) achieved this idea by constructing a liquid prism, i.e., a thinwalled glass container that is filled with the same liquid, which is then attached to the original, flat wall of the test section. Later Soloff et al. (1997) introduced the general calibration procedure which can be used to correct not only an optical distortion, but also the nonuniform magnification image of the angular-displacement method.

\subsubsection{Calibration}

Optical distortion due to inaccurate optical alignment, lens nonlinearity, and/or refraction by optical windows, fluid interfaces, and other optical elements of an experiment causes inaccuracy by introducing variable magnification. In the work presented here, a calibration suggested by Soloff et al. (1997) is used to generate an approximate mapping function. Distortion can be compensated even when the focusing aberrations cause some blurring of the images, so long as the blurring is not so severe as to make the measurement of image locations impossible or unacceptably inaccurate. The information found by the calibration can be used to do more than correct for distortion. The results of the calibration yield one or more mapping functions that provide a convenient and accurate 
way to correct for distortion in experiments when determining particle displacements.

\subsection{Error Analysis}

This section describes the error analysis of the Stereoscopic PIV system including a general error analysis, geometric error analysis and the particle size error analysis.

\subsubsection{General Stereo Error analysis procedure}

To evaluate the performance or the error of the system, it is convenient to use a fluid flow wherein the velocity at each point is exactly known a priori; however, it is difficult to control the velocities precisely through out the whole field in a real flow. Therefore, Prasad et al. (1992) and Zang and Prasad (1997) used the test photograph to simulate the elementary flows using real particles. The following is the translation test conducted by the Prasad et al. (1992): First, black carbospheres (30 $\mu \mathrm{m}$ in diameter) were mixed with white, household glue and the mixture was painted onto a $10 \mathrm{~cm} \times 12.5 \mathrm{~cm}$ piece of glass. The concentration of particle in the glue was controlled to yield an adequate density on the glass plate (about ten particles per $\mathrm{mm}^{2}$ ). After allowing the glue to dry, the surface was given a protective coat of epoxy. Next, a contact point of this glass plate was made on Kodak Technical Pan $4415(10 \mathrm{~cm} \times 12.5 \mathrm{~cm})$ film. The contact print, which displayed the particles as bright spots on a dark background, was then sandwiched between two clean glass plates and clamped onto a stage, whose movement (translation as well as rotation) could be effected in small increments, and accurately measured by means of a dial gauge. The contact print was back-lit using a 500W flood lamp and the first exposure was made. The stage was then translated horizontally by a small distance $(0.254 \mathrm{~mm})$ and a second exposure was made on the same piece of film; thus simulated

a uniformly translating flow with the vector displacement of $\Delta X=(0.254,0)$. For this study, a simple print out of the actual particles image is used. It was attached to the 
calibration plate with a translation stage to simulate a uniformly translating flow. The measurement error of the out-of-plane velocity was less than $1 \%$ throughout the entire measurement plane.

\subsubsection{Geometric Error Analysis}

The geometric error analysis for the translation system has produced relationships for estimating the individual object plane errors $\delta(\Delta x), \delta(\Delta y)$ and $\delta(\Delta z)$; however, Lawson and $\mathrm{Wu}$ (1997a) introduced a more general performance parameter by considering the ratio of the individual error components to form dimensionless groups. As shown in Figure 2.2, the angular-displacement method will be a translation method when $\theta=0^{\circ}$

Lawson and $\mathrm{Wu}$ (1997a) explains the derivation of the dimension less error quantity as follow:

Let us assume that the uncertainties in $\alpha, h, d_{o}$ and $d_{i}$ are negligible and that the errors in the image plane $\left(\Delta X_{1}, \Delta Y_{1}\right)$ and $\left(\Delta X_{2}, \Delta Y_{2}\right)$ are equal such that:

$$
\delta\left(\Delta X_{j}\right)=\delta\left(\Delta Y_{j}\right)=\delta(\Delta X)
$$

for $j=1,2$ where $\delta()$ represents the error in a given variable. Therefore using standard error analysis the uncertainties in the object planes, $\Delta x, \Delta y$ and $\Delta z$ can be written

$$
\begin{gathered}
\delta(\Delta x)=\frac{\sqrt{b_{1}^{2}+b_{2}^{2}}}{\left|a_{1} b_{2}-a_{2} b_{1}\right|} \delta(\Delta X) \\
\delta(\Delta y)=\left[\sqrt{c_{1}^{2}+c_{2}^{2}+c_{3}^{2}}\right] \delta(\Delta X) \\
\delta(\Delta x)=\frac{\sqrt{a_{1}^{2}+a_{2}^{2}}}{\left|a_{2} b_{1}-a_{1} b_{2}\right|} \delta(\Delta X)
\end{gathered}
$$


where the coefficients $c_{1}, c_{2}$ and $c_{3}$ equal

$$
\begin{gathered}
c_{1}=-\frac{2}{M_{1}+M_{2}} \\
c_{2}=-\frac{y \cos \alpha}{M_{1}+M_{2}}\left[\left(\frac{M_{1}}{d_{o 1}}-\frac{M_{2}}{d_{o 2}}\right) \frac{\sqrt{a_{1}^{2}+a_{2}^{2}}}{a_{2} b_{1}-a_{1} b_{2}}\right] \\
c_{2}=\frac{y \sin \alpha}{M_{1}+M_{2}}\left[\left(\frac{M_{2}}{d_{o 2}}-\frac{M_{1}}{d_{o 1}}\right) \frac{\sqrt{b_{1}^{2}+b_{2}^{2}}}{a_{1} b_{2}-a_{2} b_{1}}\right]
\end{gathered}
$$

Considering a major objective of stereoscopic PIV system is to obtain the overall system performance, the ratio of out-of-plane with the in-plane errors is used such that:

$$
\frac{\delta(\Delta z)}{\delta(\Delta x)}=\frac{\sqrt{a_{1}^{2}+a_{2}^{2}}}{\sqrt{b_{1}^{2}+b_{2}^{2}}}
$$

This quantity will be referred to as the error ratio $e_{r}$ and will equal

$$
e_{r}=\frac{\delta(\Delta z)}{\delta(\Delta x} \mid
$$

for any given values of $x, y$ and $z$.

If we now analyze the performance of an angular set-up with two identical cameras where $h=0, M_{1}=M_{2}=M$ and $d_{o 1}=d_{o 2}=d_{o}$, then from Equation2.13 the error ratio $e_{r}$ can be written:

$$
e_{r}=\frac{\sqrt{\cos ^{2} \alpha-\sin ^{2} \alpha\left[2\left(\frac{z}{d_{o}}\right) \cos \alpha+\left(\frac{z}{d_{o}}\right)^{2} \sin ^{2} \alpha+\left(\frac{x}{d_{o}}\right)^{2} \cos ^{2} \alpha\right]}}{\sin \alpha \sqrt{1+2\left(\frac{z}{d_{o}}\right) \cos \alpha+\left(\frac{z}{d_{o}}\right)^{2} \sin ^{2} \alpha+\left(\frac{x}{d_{o}}\right)^{2} \cos ^{2} \alpha}} \mid
$$


At the center of the measured field where $x=0, y=0, z=0$, the error ratio will simply equal:

$$
e_{r}=\frac{1}{\tan \alpha} \mid
$$

This relationship is plotted in Figure 2.4. The optimum angle for the angular method suggested by Lawson and $\mathrm{Wu}$ (1997b) was between $\alpha=20^{\circ}$ to $\alpha=30^{\circ}$ which corresponds to the error ratio of 2.75 to 1.73 respectively. By enforcing the Scheimpflug condition, the angle can be set further apart for better accuracy without distorting the particle image. At $\alpha=40^{\circ}$ the error ratio can be further reduced to 1.19.

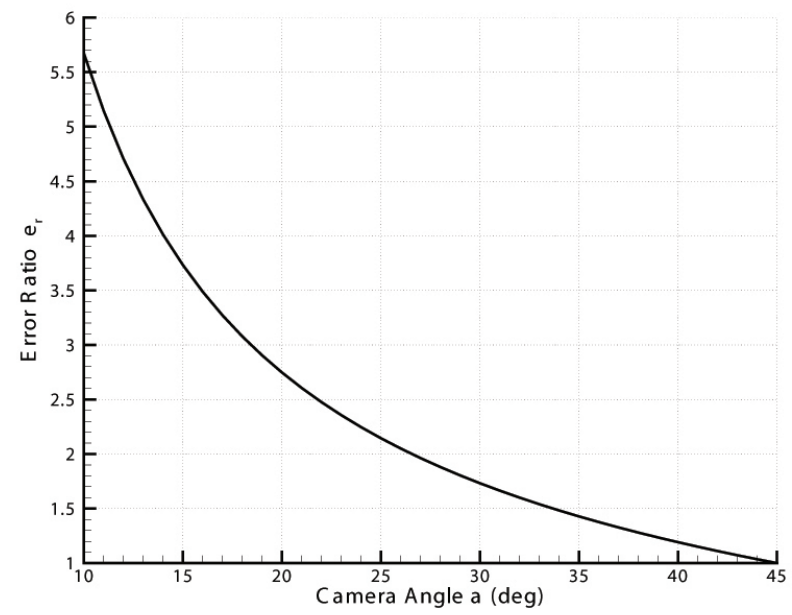

Figure 2.4: Error ratio versus camera angle for angular-displacement method

\subsubsection{Particle size}

According to Prasad et al. (1992) the particle image diameter strongly influence accuracy through two distinct error mechanisms. When the particle image is small compared to the pixel size, mean bias error becomes significant due to finite numerical resolution of the correlation function. Conversely, when the particle image is large, random error due to irregularities in the electronic images predominates. They quantified 
the error by defining a ratio of diameter of the particle image $\left(d_{\tau}\right)$ to the pixel spacing $\left(d_{p i x}\right)$ i.e., number of pixels per particle $\left(\frac{d_{\tau}}{d_{p i x}}\right)$. From the particle image simulation the

bias error diminishes rapidly for larger image diameters, after $\left(\frac{d_{\tau}}{d_{p i x}}\right) \approx 2$. Although larger particles do result in reduced bias errors, the random error is directly proportional to the absolute size of the particle image, $\left(d_{\tau}\right)$. By taking the sum of the squares of the bias and random errors, Prasad et al. (1992) determined the optimum ratio of $\left(\frac{d_{\tau}}{d_{p i x}}\right)$ to be $2.2 \sim 2.7$ where total error was minimized.

\subsection{Experiment Setup}

The following section describes the stereoscopic imaging system and data acquisition methods used to obtain the experimental PIV data. A general in-situ calibration procedure was conducted in the present study to obtain the mapping functions between the image planes and object planes as described in section 2.1.5.

\subsubsection{Wind Tunnel and Stereoscopic PIV Set up}

The experimental study was conducted in a closed-circuit, low-speed wind tunnel located in the Aerospace Engineering Department of Iowa State University as shown in Figure 2.5. The tunnel has a test section with a $12.0 \mathrm{in} \times 12.0 \mathrm{in}(30.5 \mathrm{~cm} \times 30.5 \mathrm{~cm})$ cross section and the walls of the test section are optically transparent. The tunnel has a contraction section upstream of the test section with honeycombs, screen structures and a cooling system installed ahead of the contraction section to provide uniform low turbulent incoming flow into the test section. The standard deviation of velocity fluctuations at the test section entrance was found to be about $0.8 \%$ of the free-stream velocity, measured by using a hotwire anemometer.

The test model used in the present study was a zero-sweep, untwisted half wing, with a NACA0012 symmetric profile. The wing had a rectangular planform with a semi-span 


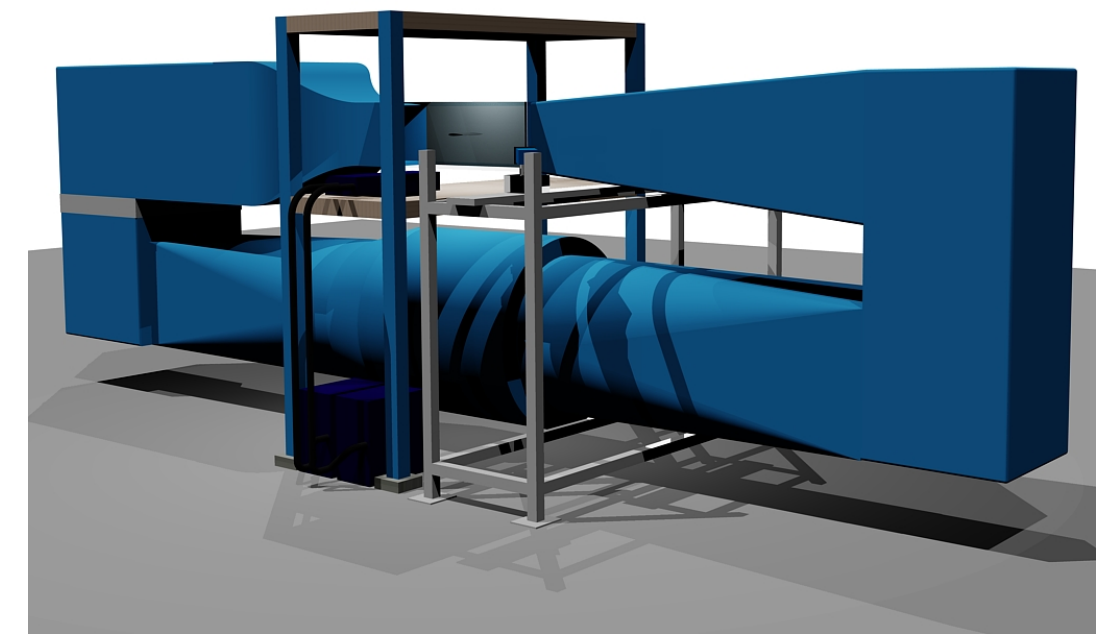

Figure 2.5: Low speed wind tunnel used in the experiment.

$\mathrm{b}=6$.0in and a chord $\mathrm{c}=4.0 \mathrm{in}$ and a plane tip with sharp edges. Its root was mounted on one side of the wind tunnel wall as shown in Figure 2.6. The angle of attack of the tested wing could be changed using a rotary table mounted outside the wind tunnel with the axis of rotation passing through the airfoil's aerodynamic center, or quarter-chord point. The wing was mounted through the wind tunnel wall such that its quarter-chord axis was along the centerline of the wind tunnel. Also the wing was on the traverse system on the wall, so that the wing could translate in the stream-wise direction. The traverse system is used to move the wing so that SPIV measurements are taken at various down stream location without moving the laser and optics. Following the work of Bailey \& Tavoularis (2008), a boundary layer trip wire was attached on the suction surface of the airfoil, 0.10c away from the leading edge, to induce transition, thus reducing the possibility of separation and sensitivity to free-stream conditions.

For the wall-effect experiments, the additional U-shaped walls are inserted in the test section to reduce the distance between the wing tip and the tip-side wall. The width 


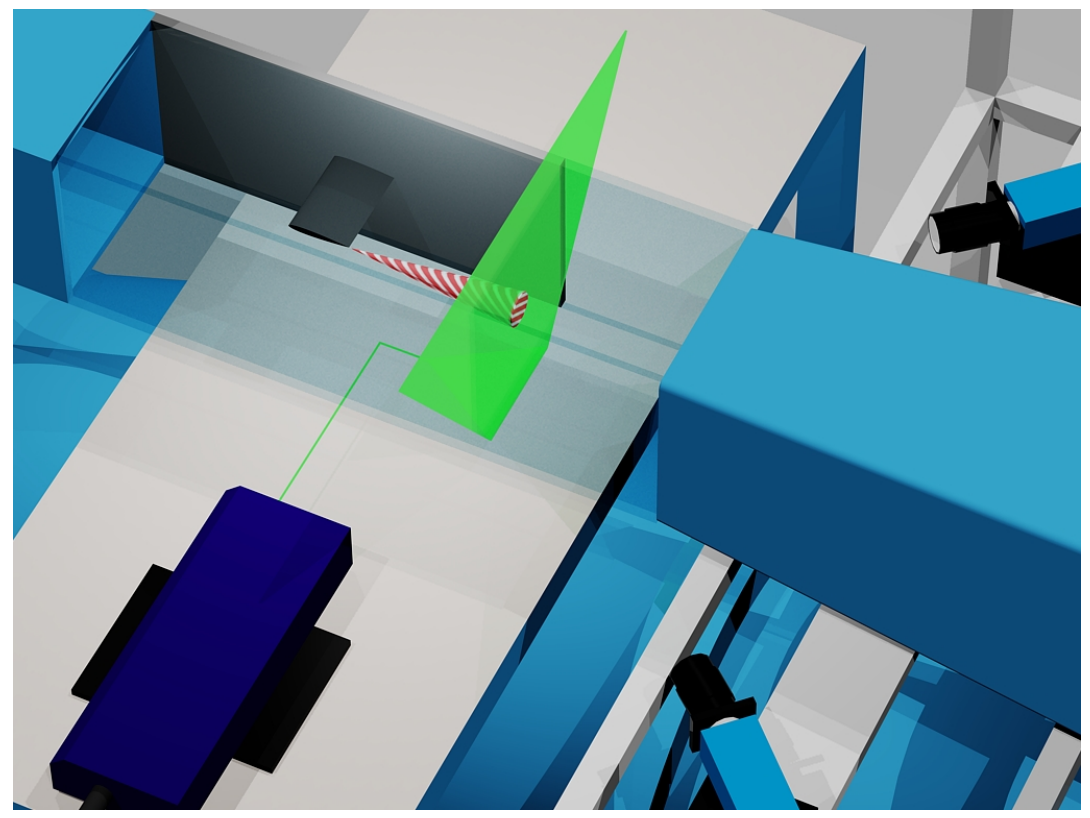

Figure 2.6: The wing mount and laser position (optics not shown).

of the wall, the parameter a shown in Figure 2.7b, is varied to 2.0, 4.0 and 5.0 in so that the distance between the wing tip and wall is set to 4.0, 2.0 and 1.0 in respectively. These additional walls are made of optically transparent acrylic plates with a thickness of $1 / 8$ in. This wall is thick enough to stand rigid in the test section; yet, thin enough to cause no tunnel blockage issues. The index of refraction varies due to the additional wall and also the location of the wall so that the in-situ calibration procedure is performed each time to compensate the optical distortion. The stereo PIV measurements for the wall-effect are taken at 4 chord lengths downstream with AOA of 5.0 and 10.0deg. Data is processed in the same way as the no additional wall cases.

\subsubsection{Illumination and Hardware}

Illumination for the present study is provided by a double-pulsed Nd:YAG laser (NewWave Gemini 200) adjusted on the second harmonic and emitting two pulses of $200 \mathrm{~mJ}$ at the wavelength of $532 \mathrm{~nm}$ with a repetition rate of $4.0 \mathrm{~Hz}$. The laser beam 


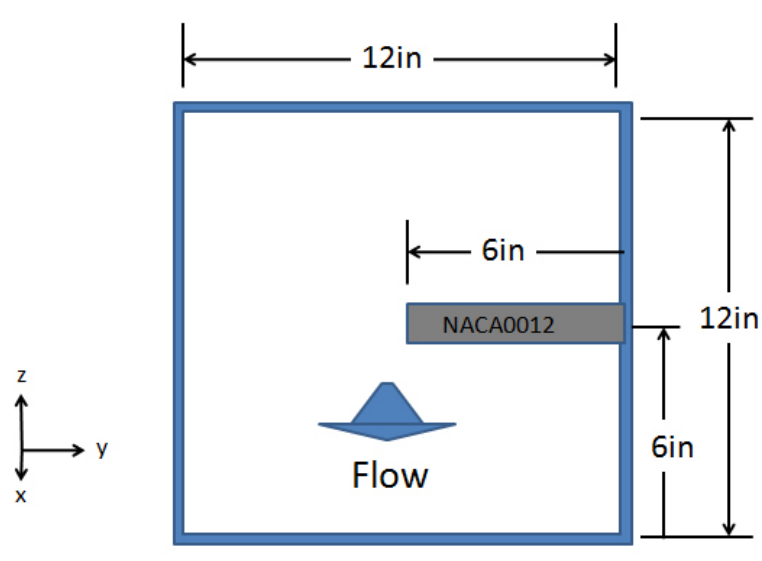

(a) Without wall

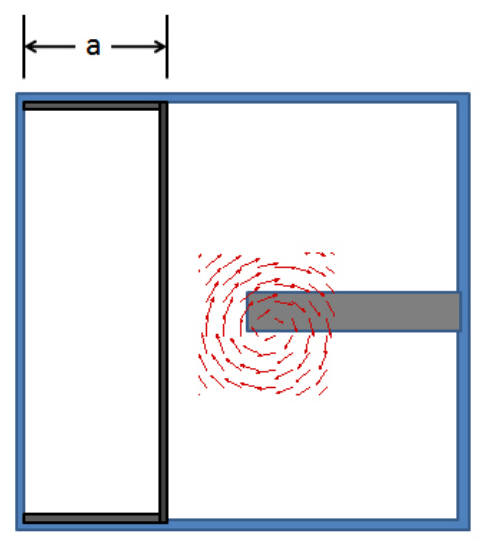

(b) With additional inner wall

Figure 2.7: Cross sectional view of wind tunnel test section.

was shaped to a sheet by a set of mirrors, spherical and cylindrical lenses. Later this set of optics are replaced by the LaVision VZ-Beam Steering Arm. The setup configuration is shown in Figure 2.6. The thickness of the laser sheet in the measurement region is about $2.0 \mathrm{~mm}$. Two high-resolution 12-bit (1600 x 1200 pixel) CCD camera (PCO1600, CookeCorp) equipped with Ai AF Micro-Nikkor 60mm f/2.8D lens were used to perform stereoscopic PIV image recording. The two CCD cameras were arranged in an angulardisplacement method configuration to get a large overlapped view. With the installation of tilt-axis mounts, the lenses and camera bodies were adjusted to satisfy the Scheimpflug condition as described in section 2.1.3. The $f \#$ is set to $f / 8.0$ to give enough focal depth considering with the particle size on CCDs. 


\section{CHAPTER 3. RESULTS}

This chapter describes the results of the measurements taken by the stereoscopic PIV system described in Section 2.3. The behavior of the vortex is presented with three different effects. They are an angle of attack effect, downstream effect, and wall effect. Each effect is analyzed by a wandering amplitude plot, vorticity plot, vortex radius plot, and contour plots of T.K.E. and Reynolds Stress for vortex re-centered data and before re-centering data. In this study, the vortex center is defined as the point which has the zero velocity $(v=w=0.0)$. Please refer to the section 1.4 for more details about the vortex center.

\subsection{AOA Effect}

In this section, the angle of attack effect on vortex behavior is described. During the experiment, the angle of attack is changed from 0.0 to 14.0 degrees at 2.0 degrees increments but the results are shown from 2 degrees to 12 degrees. 0.0 and 14.0 degrees data is excluded from the results because no vortex generation at 0.0 degrees of angle of attack due to the symmetric airfoil, and the airfoil is in a stall condition at 14.0 degrees.

Figure 3.1a shows the angle of attack effect on the wandering amplitude. The circle symbol indicates the wandering amplitude in y-direction, $\sigma_{y}$, and the square symbol indicates the amplitude in z-direction, $\sigma_{z}$. As shown in Figure 3.1a, the both amplitudes, $\sigma_{y}$ and $\sigma_{z}$, are decreasing with increasing the angle of attack till $\alpha=6.0^{\circ}$. After $\alpha=6.0^{\circ}$, 
the wandering amplitudes stay constant around $\sigma=0.4 \mathrm{~mm}$. This result closely agrees with the study done by Devenport et al. (1996). They got the wandering amplitudes $(\sigma / c)$ of $0.009,0.006,0.006$, and 0.005 at $\alpha=2.5,3.9,5.0$ and $7.5^{\circ}$ respectively, where the current study shows the wandering amplitude of $0.009,0.005,0.004$, and 0.003 at $\alpha=2.0,4.0,6.0$ and $8.0^{\circ}$ respectively. This proves that the method which predicts the wandering amplitude from the measured Reynolds stresses at the center, suggested by Devenport et al. (1996) and shown in section 1.2, is fairly accurate. Also, as shown by the Devenport et al. (1996) study, the vortex wanders $0.002 \mathrm{c}$ or $0.001 \mathrm{c}$ more in ydirection (spanwise direction). This trend can be seen in the current study as the vortex wanders $0.002 \mathrm{c}$ or $0.004 \mathrm{c}$ more in the spanwise direction. As shown in the Table 3.2, the wandering is found to have the largest amplitude when the vortex had the lowest peak tangential velocity. When the tangential velocity, $v_{\theta}$, is $2.0 \mathrm{~m} / \mathrm{s}$ at $\alpha=2.0^{\circ}$, the vortex wanders about $0.9 \mathrm{~mm}$ in both direction but when the angle of attack is increased to $12.0^{\circ}$, the tangential velocity is increased to $8.60 \mathrm{~m} / \mathrm{s}$ and the vortex wandering is reduced by $70 \%$. For the same flow condition, even though the wandering is constant at the higher angle of attack, the increase in the tangential velocity will have some effect on decreasing the vortex wandering which agree with the finding by Bailey and Tavoularis (2008).

The Figure 3.1b shows the angle of attack effect on the vorticity at the center of the vortex. The Figure 3.1b suggests that the vorticity increases negatively with respect to the angle of attack. This makes sense since the wing-tip vortex is induced by the lift of the $3 \mathrm{D}$ wing. When the AOA is increased the lift will be increased, hence the vorticity increases or vice versa. The notable point of this plot is that the vorticity stays constant between $\alpha=6.0^{\circ}$ and $8.0^{\circ}$. The vortex strength and the wandering amplitude are correlated and shown by Heyes et al. (2004). Their finding was that a linear decrease in wandering amplitude with increasing vortex strength which does not match with the current study. As shown in Table 3.2, the vortex wandering decrease with increasing 


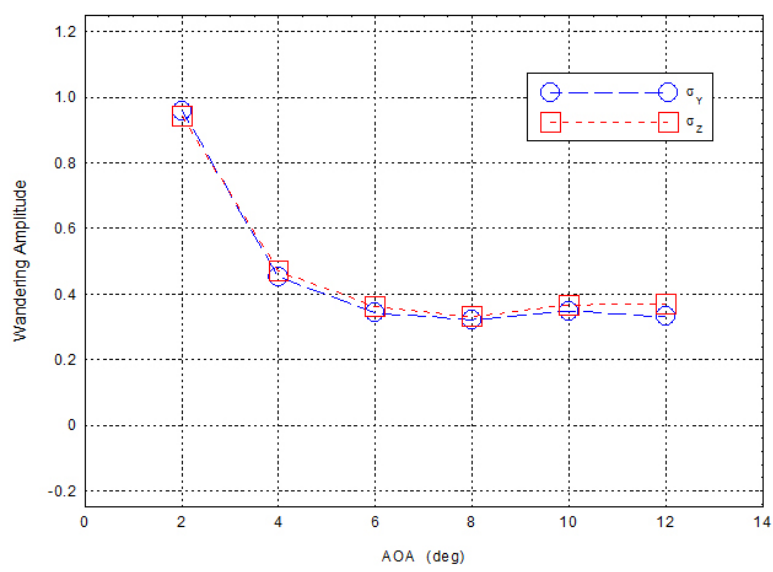

(a) Wandering amplitude.

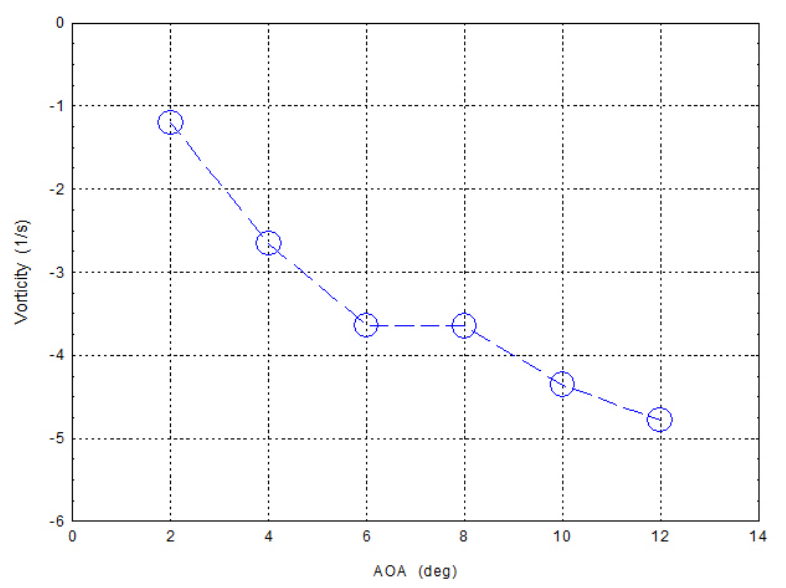

(b) Vorticity at vortex center.

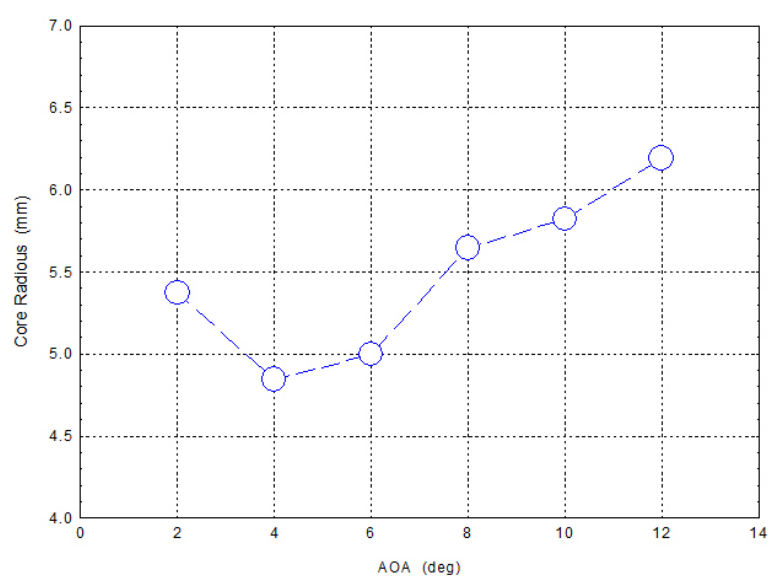

(c) Vortex core radius.

Figure 3.1: AOA effect.

vorticity that agree with Heyes et al. (2004) up to $\alpha=6.0^{\circ}$, but after $8.0^{\circ}$, the vortex wandering amplitudes stay constant where the vorticity increases linearly. This suggests that the mechanism responsible for wandering is not self induced as proposed by Rokhsaz et al. (2000), in which case it would be expected that wandering would increase with vortex strength. Also, the vortex wandering is independent of the vortex strength, as shown by the current study, and that there is a constant wandering amplitude region with increase in the vorticity. This result further suggests that the vortex is responding to an external influence, for example free-stream turbulence or fluttering of the wing in 
the test section.

Figure 3.1c shows the angle of attack effect on the vortex core radius. Initially the axial-averaged vortex core radius is $5.37 \mathrm{~mm}$ at $\alpha=2.0^{\circ}$. When the angle of attack is increased to $\alpha=4.0^{\circ}$, the vortex core radius gets smaller to $4.85 \mathrm{~mm}$. As the angle of attack gets larger toward $\alpha=12.0^{\circ}$, the core radius gets bigger. At $\alpha=6.0^{\circ}$, the vortex core radius is $5.0 \mathrm{~mm}$ and for the larger angle of attack, at $\alpha=12.0^{\circ}$, the vortex radius is increased by $20 \%$ to $6.19 \mathrm{~mm}$. This trend, a small decrease in the vortex core before the increasing core radius at high angle of attack, also can be seen in the study done by the Gerontakos and Lee (2006). By using a sweptback and tapered NACA0015, Gerontakos and Lee (2006) showed the sharp decrease in the core radius up to $\alpha=6.0^{\circ}$ and the gradual increase as the angle approaches to $9.0^{\circ}$.

Figures 3.2 and 3.3 show the normalized T.K.E. distribution of the vortex with and without the wandering correction. The left column of the figures show the distribution without center correction and the right column show the distribution with center correction. At $\alpha=2.0^{\circ}$, the highest T.K.E. value of about 0.0016 is observed at the center of the vortex (Figure 3.2a). After tracking the vortex center in every frame to eliminate the vortex wandering effect, as shown in Figure 3.2b, the T.K.E. at the vortex center is reduced to less than 0.0001. By comparing the two plots, it can be said that the higher T.K.E. values in the center of the vortex is mainly due to the vortex wandering. As the angle of attack increases, the spiral wake becomes larger but the maximum T.K.E. value in the wake stays about 0.0010 . From increasing the angle of attack from $8.0^{\circ}$ to $10.0^{\circ}$, the T.K.E. increases significantly. At $\alpha=8.0^{\circ}$, the maximum T.K.E. is about 0.0016 at the center but for $\alpha=10.0^{\circ}$, the T.K.E. goes above 0.020 and fills most of the vortex core. Even after removing the wandering, the T.K.E. in the vortex core increases from 0.003 to 0.006 (Figure $3.3 \mathrm{~b}$ and $3.3 \mathrm{~d}$ ). This is suggesting that the vortex core is much more turbulent at higher angles of attack and non re-centered T.K.E. plots at higher angles of attack (Figure 3.3c and 3.3e) contain the turbulence from the vortex wandering 
and also from the turbulence within the vortex core as well.

Figures 3.4 and 3.5 show the Reynolds Stress distribution of the vortex before and after correcting the center. The left column of the plots shows the distribution without center correction and the right column shows the distribution with center correction. Before correcting the center, the vortex core has two pairs of positive and negative Reynolds Stress except for at $\alpha=2.0^{\circ}$ and $\alpha=10.0^{\circ}$. At $\alpha=2.0^{\circ}$, the positive stress is dominant and at $\alpha=10.0^{\circ}$, the negative stress is dominant. These pairs of stresses become stronger as the angle of attack increases. For $\alpha=4.0^{\circ}$ (Figure 3.4c), the maximum stresses are about \pm 1.5 and when the angle of attack is increased to $\alpha=12.0^{\circ}$, as shown in Figure 3.5e, the stress level exceeds \pm 3.0 . These two pairs of positive and negative stresses are found in the study done by Chow et al. (1997). They describe that the contours of the $\overline{v^{\prime} w^{\prime}}$ stress in the wake planes had a four-leaf clover pattern with alternately changing sign of stresses in each leaf. Each leaf was roughly aligned at $\pm 45^{\circ}$ off the $\mathrm{y}$ and $\mathrm{z}$ axis, with positive levels of stress found in the first and third quadrants $(+/+$ and - values of $\mathrm{z}$ and $\mathrm{y}$, respectively) and negative levels of stress found in the second and fourth quadrants. According to Chow et al. (1997), this four-leaf clover pattern is explained by converting the Reynolds stress equation from a Cartesian coordinate to the cylindrical coordinate system as shown below.

$$
\overline{v^{\prime} w^{\prime}}=\left(\overline{v_{r}^{\prime 2}}-\overline{v_{\theta}^{\prime 2}}\right) \cos \theta \sin \theta+\left(\cos ^{2} \theta-\sin ^{2} \theta\right)
$$

Eqn.3.1 shows show a $\pm 45^{\circ}$ orientation of the $\overline{v^{\prime} w^{\prime}}$ stress implies that the $v_{r}^{\prime 2}>v_{\theta}^{\prime 2}$ and that $\overline{v_{r}^{\prime 2}}, \overline{v_{\theta}^{\prime 2}} \gg \overline{v_{r}^{\prime} v_{\theta}^{\prime}}$.

The right columns of Figures 3.4 and 3.5 show the Reynolds stress distribution after the wandering correction. As shown in the figures, the Reynolds Stresses in the core region cannot be seen after the wandering correction. For the lower angles of attack, the stress level is near 0.0003 before the correction but after the wandering correction, the stress is completely neutralized to less than 0.000015. For the higher angles of attack, 
especially $\alpha=10.0^{\circ}$ and $12.0^{\circ}$, some stress is still remaining even after the correction; however, the stress level is down by more than $50 \%$, from 0.0003 or greater to about 0.00015. This reduction in the stresses tells that the four-leaf clover pattern in the vortex core is mainly due to the vortex wandering. Chow et al. (1997) described the mathematics behind the stress pattern but the current study suggests that the wandering is strongly influencing the $v_{r}^{\prime 2}>v_{\theta}^{\prime 2}$ and $\overline{v_{r}^{\prime 2}}, \overline{v_{\theta}^{\prime 2}} \gg \overline{v_{r}^{\prime} v_{\theta}^{\prime}}$ conditions. Interestingly enough, the wandering correction diminished the stress levels within the core and only within the core. For example, at $\alpha=6.0^{\circ}$, the stress pairs only within the core disappeare and the stresses within the spiral wake, especially the square shaped positive stress region, stay the same. It can be said that the Reynolds Stress outside of the vortex core is independent of the vortex wandering and the stresses in the wake spiral is induced by perhaps the vortex roll-up. 


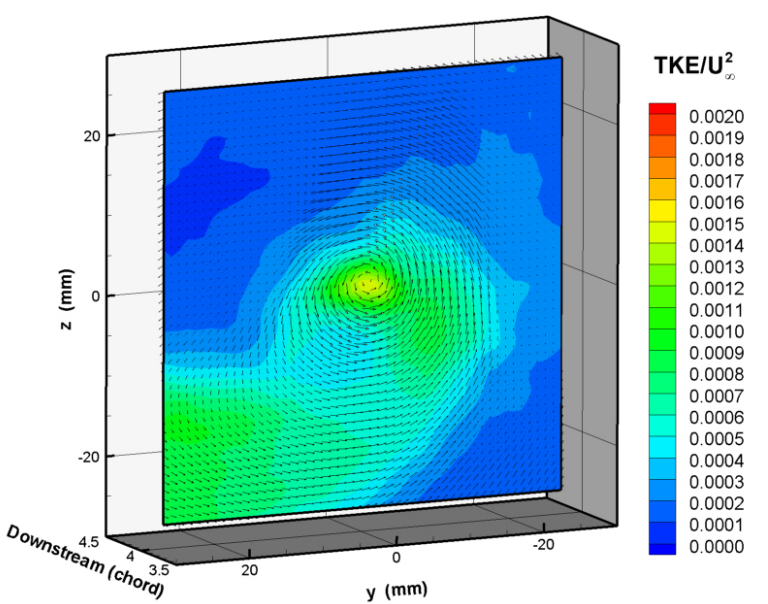

(a) $\alpha=2.0^{\circ}$

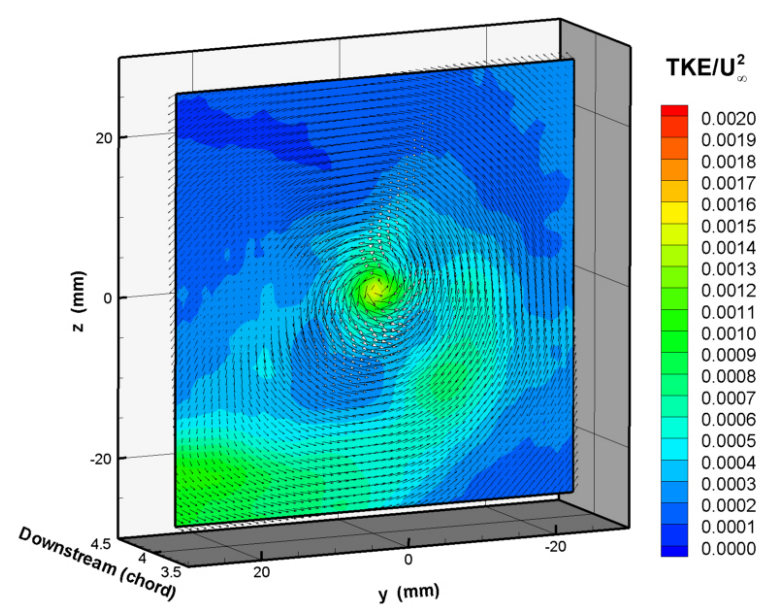

(c) $\alpha=4.0^{\circ}$

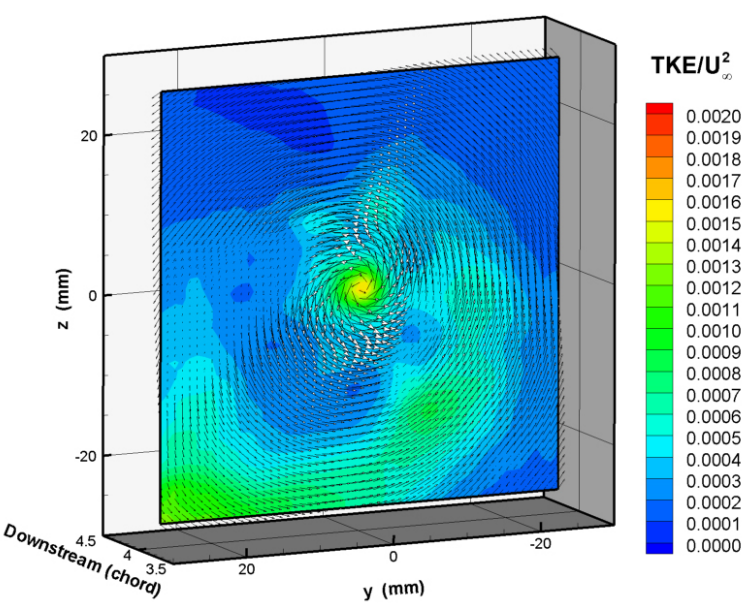

(e) $\alpha=6.0^{\circ}$

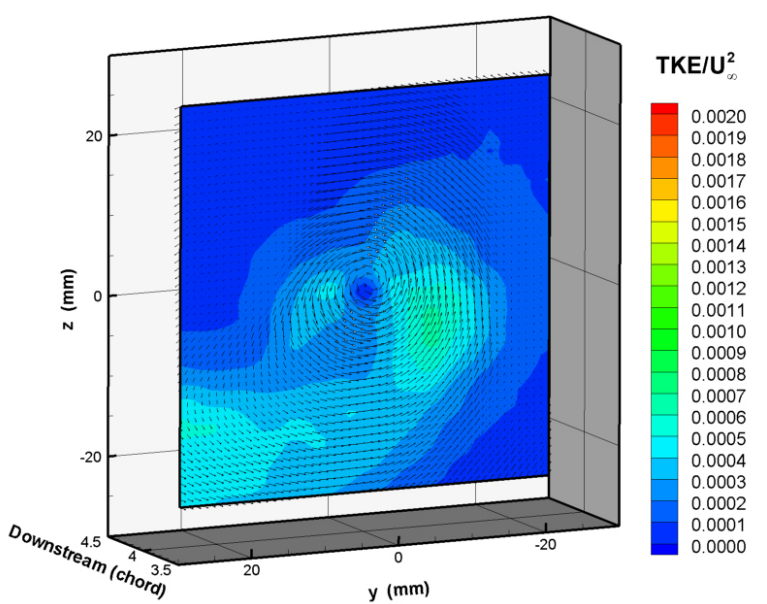

(b) $\alpha=2.0^{\circ}$, re-centered

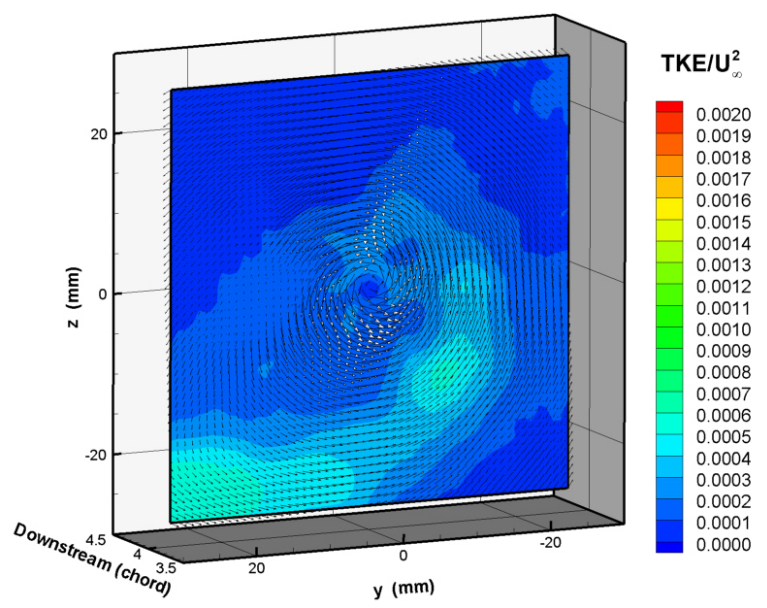

(d) $\alpha=4.0^{\circ}$, re-centered

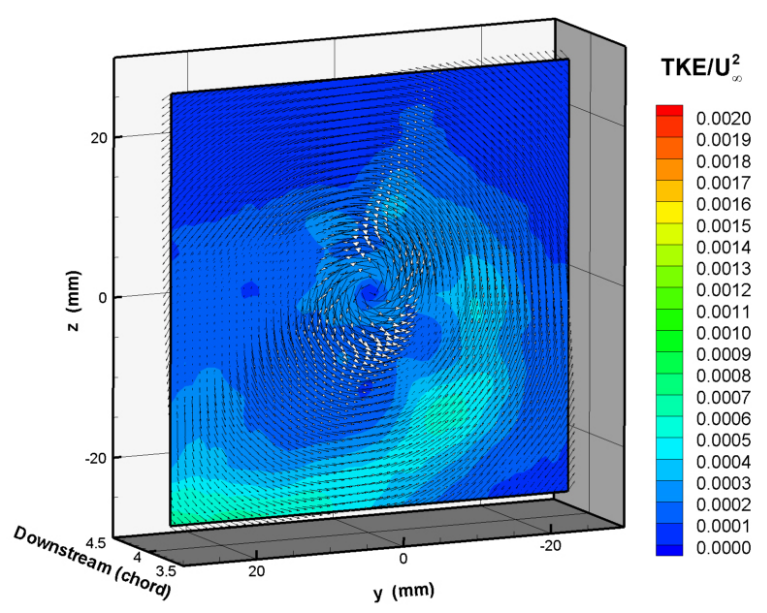

(f) $\alpha=6.0^{\circ}$, re-centered

Figure 3.2: Normalized T.K.E. distributions for lower angle of attack. 


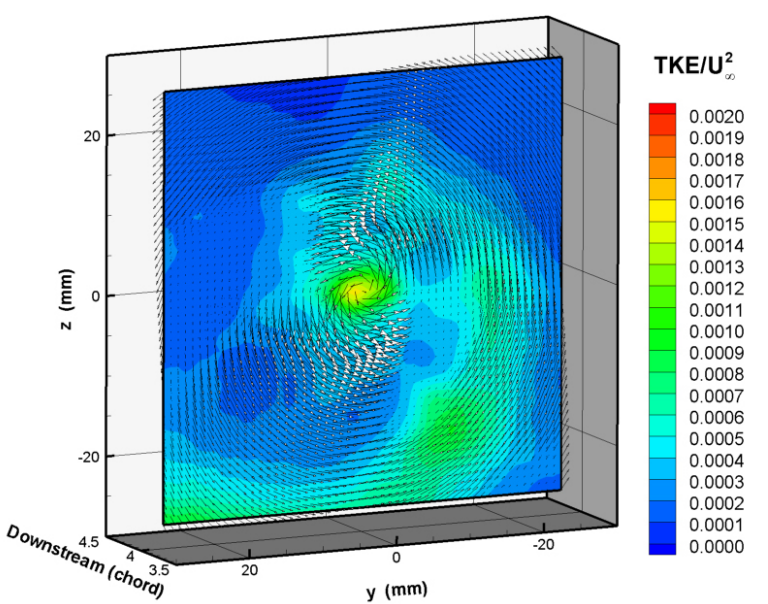

(a) $\alpha=8.0^{\circ}$

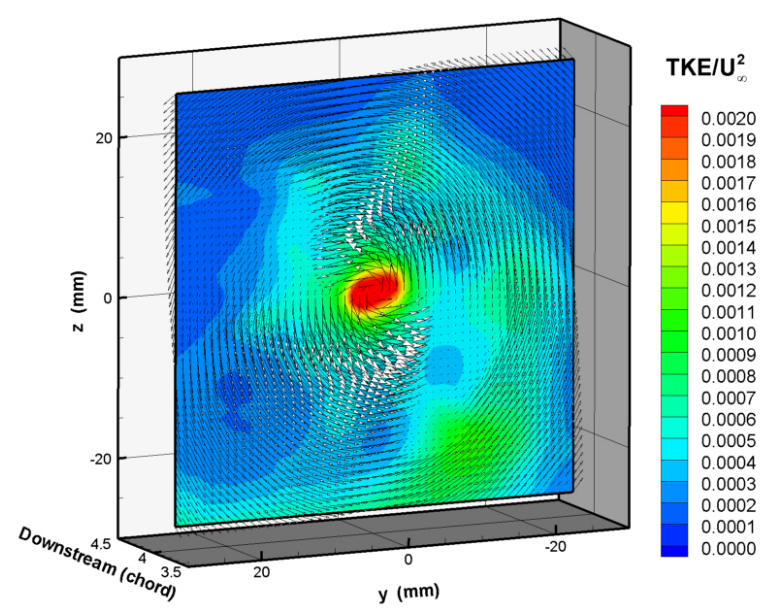

(c) $\alpha=10.0^{\circ}$

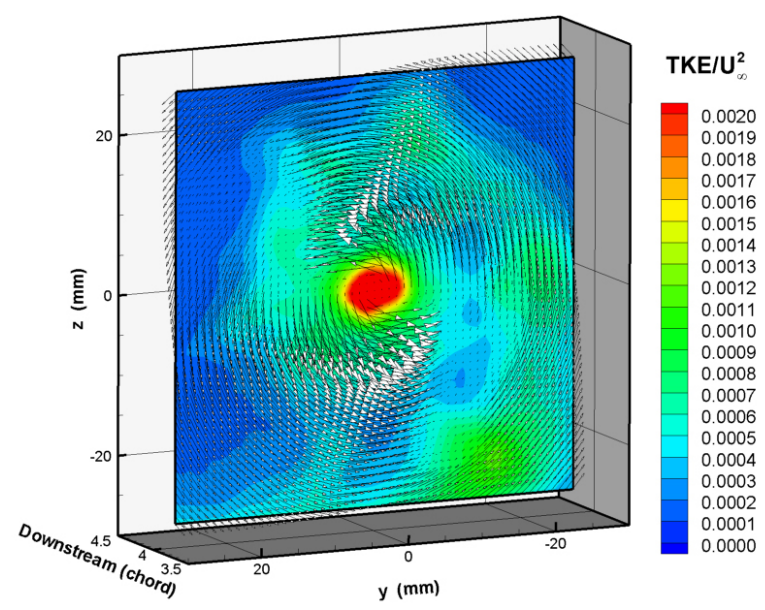

(e) $\alpha=12.0^{\circ}$

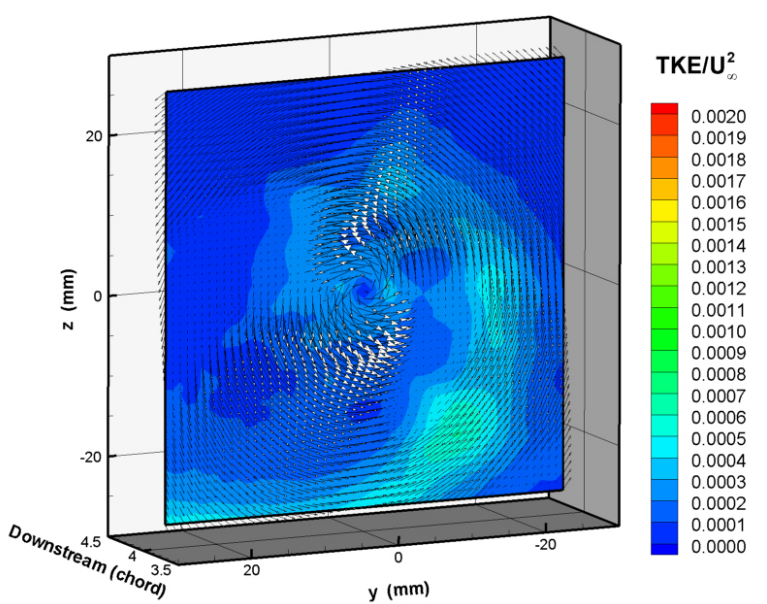

(b) $\alpha=8.0^{\circ}$, re-centered

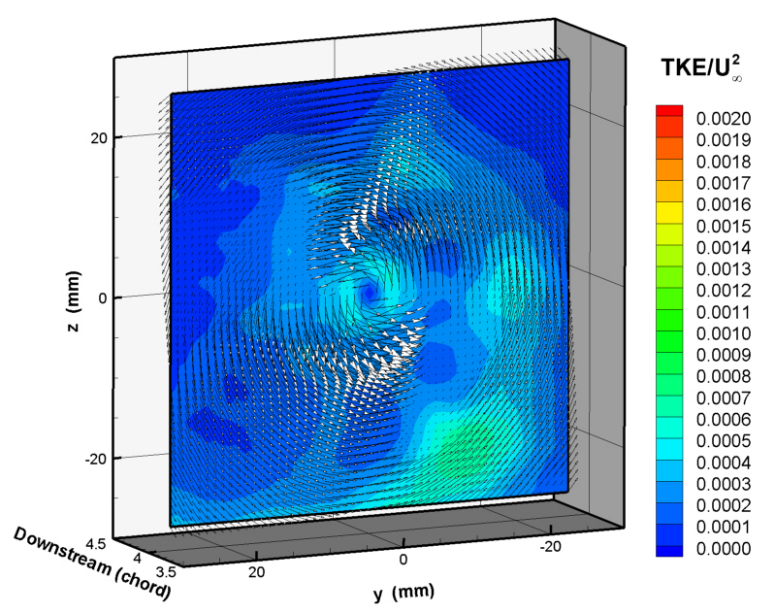

(d) $\alpha=10.0^{\circ}$, re-centered

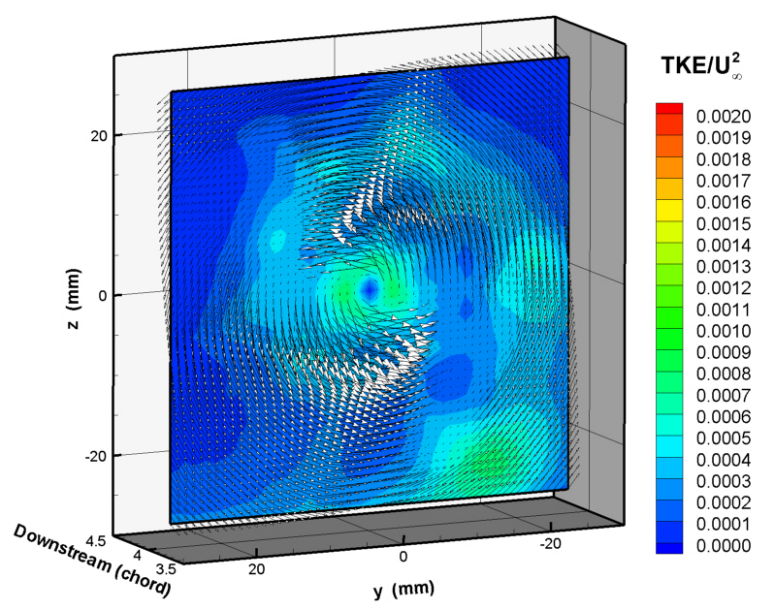

(f) $\alpha=12.0^{\circ}$, re-centered

Figure 3.3: Normalized T.K.E. distributions for higher angle of attack. 


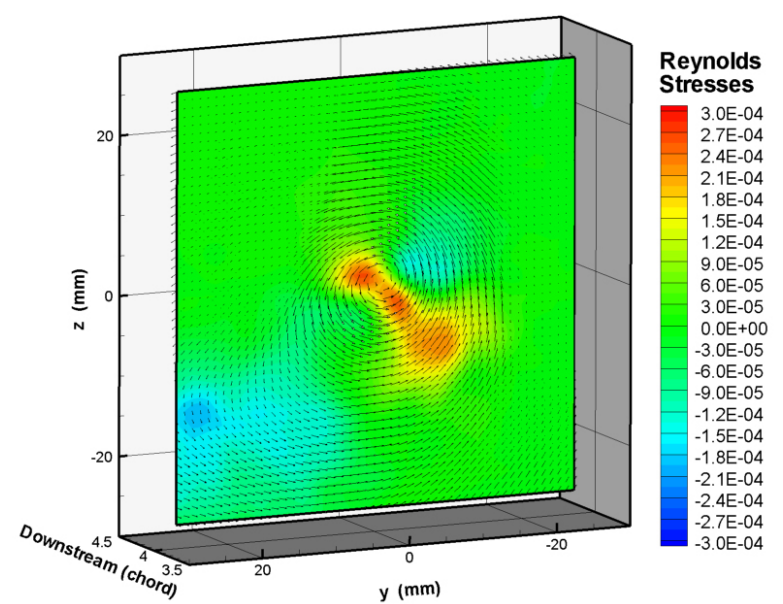

(a) $\alpha=2.0^{\circ}$

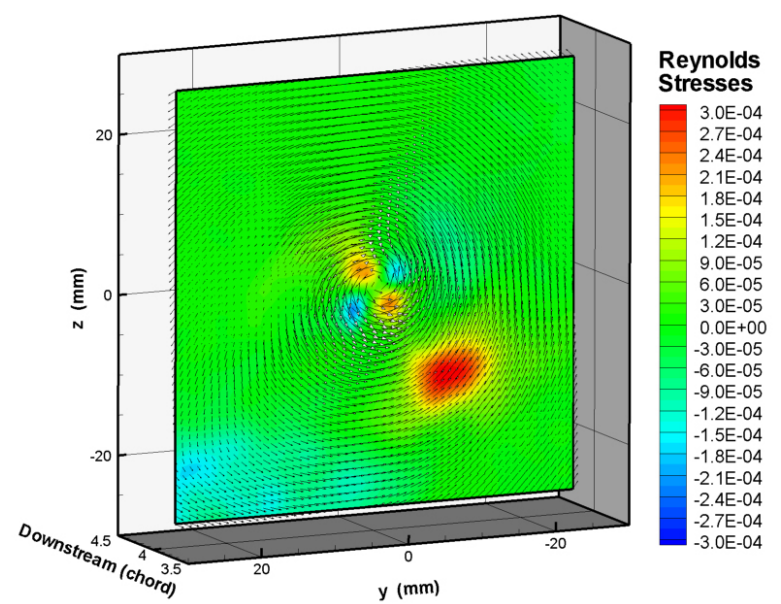

(c) $\alpha=3.0^{\circ}$

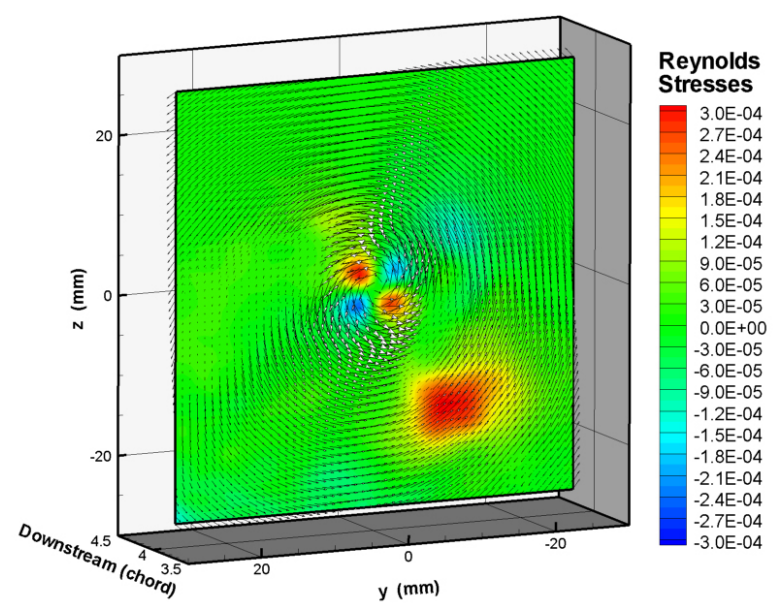

(e) $\alpha=6.0^{\circ}$

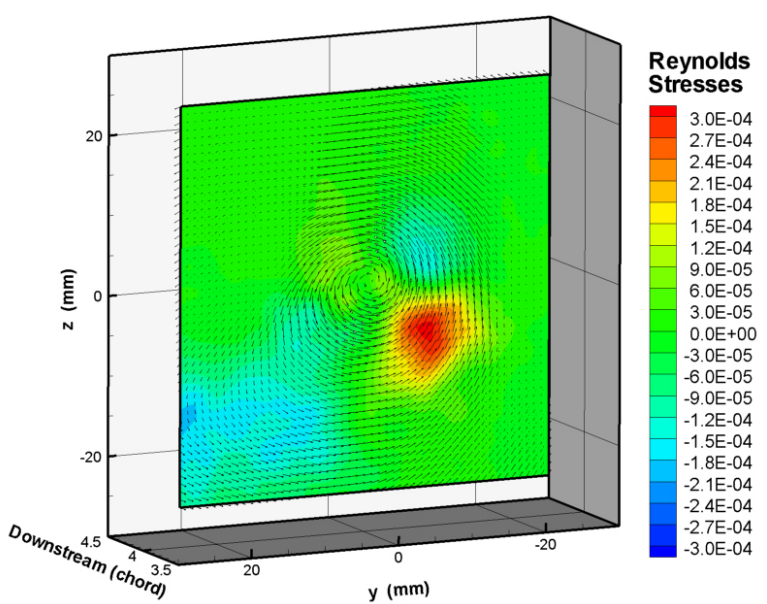

(b) $\alpha=2.0^{\circ}$, re-centered

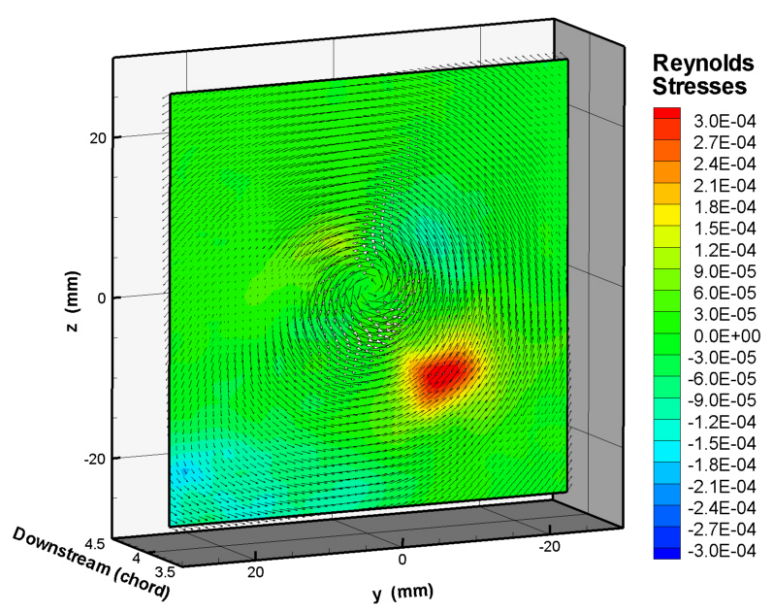

(d) $\alpha=3.0^{\circ}$, re-centered

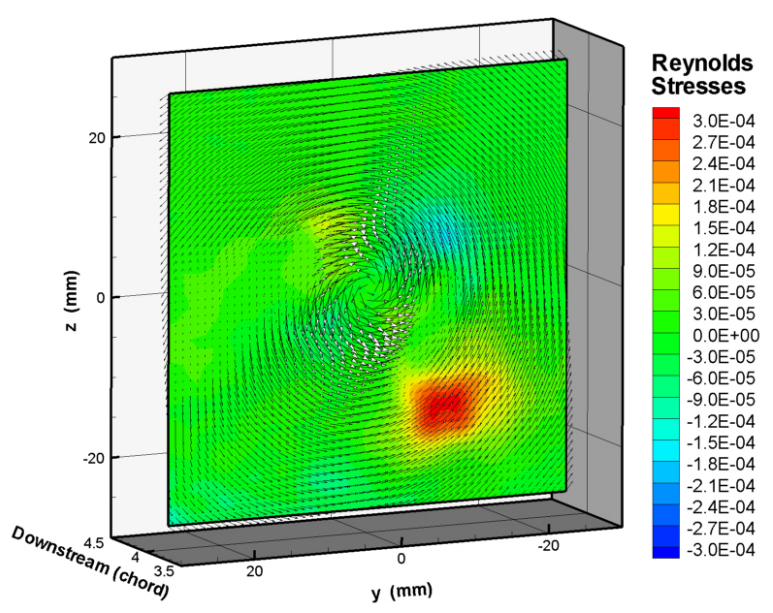

(f) $\alpha=6.0^{\circ}$, re-centered

Figure 3.4: Normalized Reynolds stress distributions for lower angle of attack. 


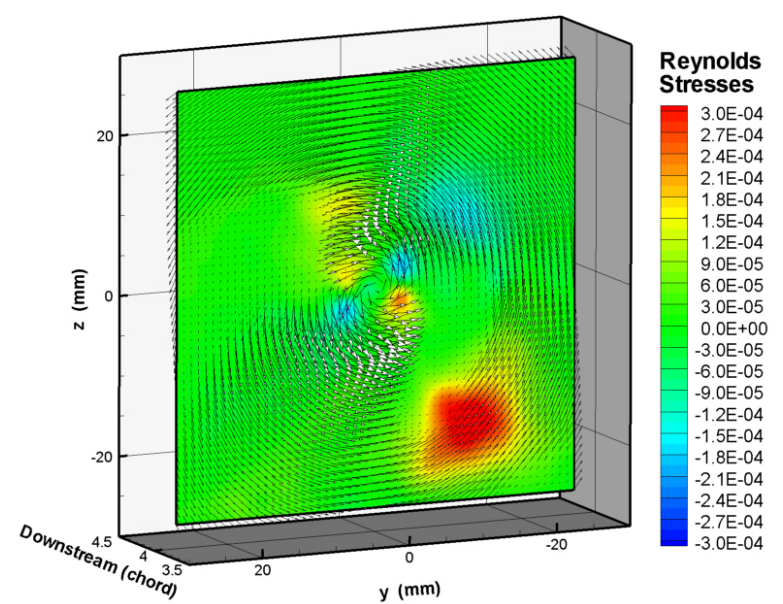

(a) $\alpha=8.0^{\circ}$

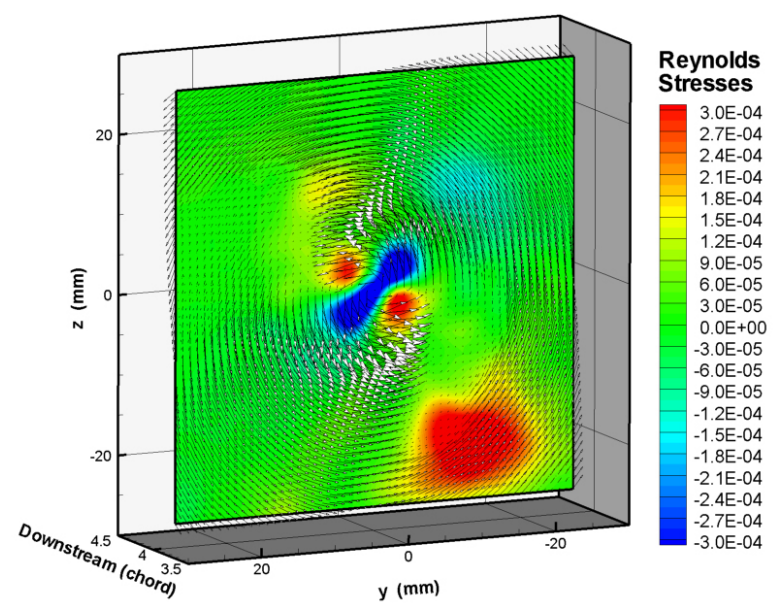

(c) $\alpha=10.0^{\circ}$

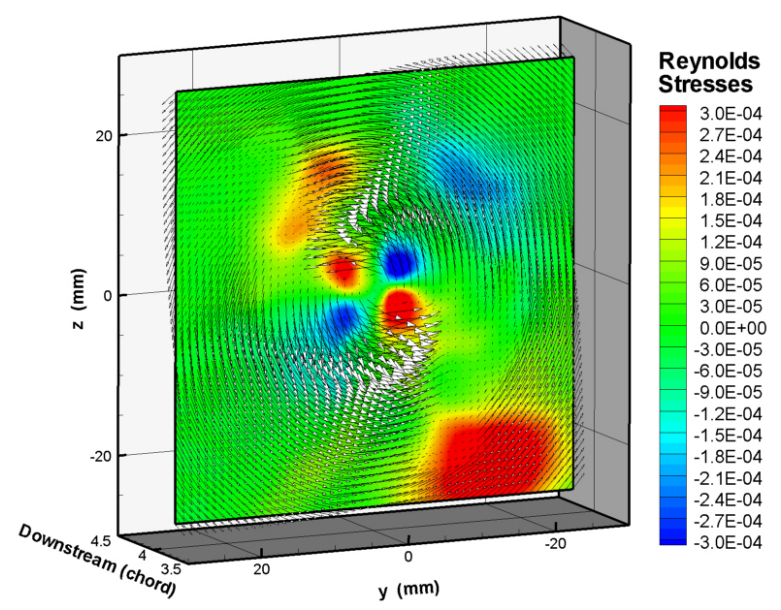

(e) $\alpha=12.0^{\circ}$

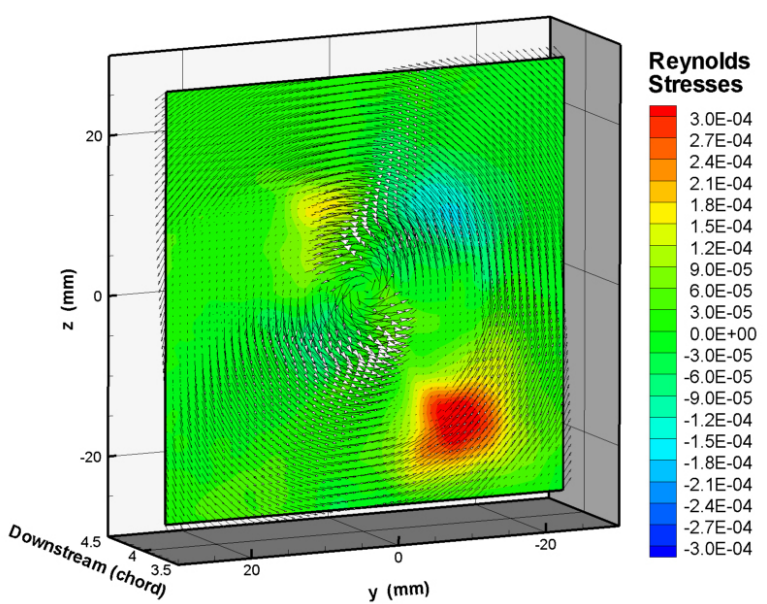

(b) $\alpha=8.0^{\circ}$, re-centered

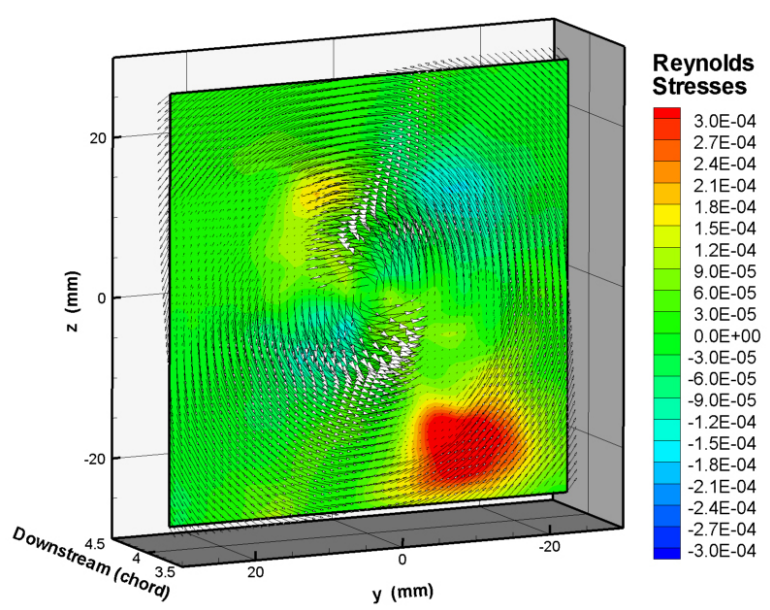

(d) $\alpha=10.0^{\circ}$, re-centered

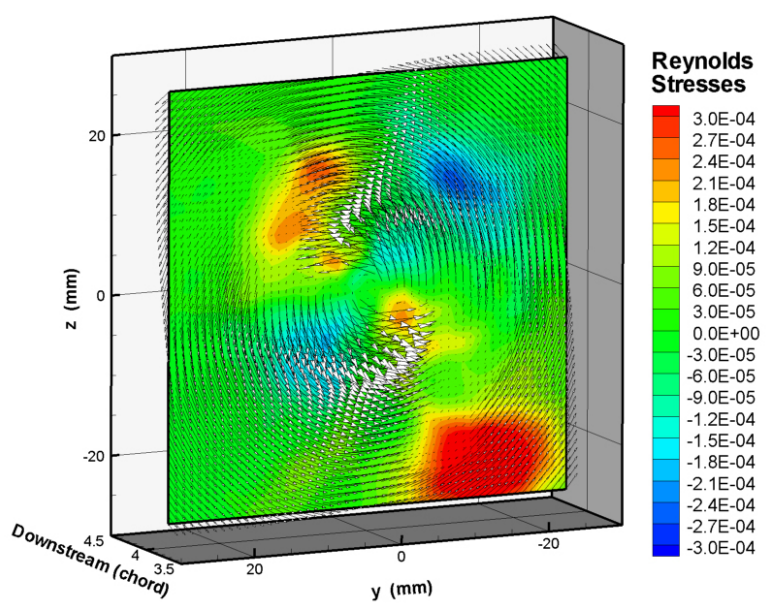

(f) $\alpha=12.0^{\circ}$, re-centered

Figure 3.5: Normalized Reynolds stress distributions for higher angle of attack. 


\subsection{Downstream Effect}

This section describes the downstream effect on the vortex behavior in the wind tunnel. SPIV measurements are taken at 1.0, 2.0, 3.0, and 4.0 chord lengths downstream of the wing with 5.0 and 10.0 degree angles of attack. Figure 3.6a shows the downstream effect on wandering amplitudes. The circle symbols are indicating the wandering amplitude at $\alpha=5.0^{\circ}$, and square symbols are indicating the amplitude for $\alpha=10.0^{\circ}$. For both angles of attack, the solid lines are indicating the wandering amplitude in $\mathrm{y}$-direction, $\sigma_{y}$, and dashed lines are indicating the amplitude in z-direction, $\sigma_{z}$. For $\alpha=5.0^{\circ}$, the wandering amplitude starts from about $\sigma=0.6 \mathrm{~mm}$ at 1.0 chord length downstream and as the vortex travels down to four chord lengths downstream and the wandering amplitudes become smaller to $0.4 \mathrm{~mm}$. By looking at solid and dashed lines, the gap between the two lines becomes smaller from 1.0c to 4.0c downstream indicating that the wandering direction becomes axisymmetric as the vortex travels downstream. For $\alpha=10.0^{\circ}$, the wandering amplitudes become smaller between the 1.0c and 2.0c downstream from $\sigma=0.5 \mathrm{~mm}$ to $0.4 \mathrm{~mm}$ and they stay the same, about $\sigma=0.4 \mathrm{~mm}$, for further downstream. For the directionality, the vortex wanders in y-direction more right after leaving the trailing edge of the wing, and it wanders z-direction more for further downstream. This cross over occurs between 2.0c to 3.0c. Figure 3.6b shows the downstream effect on vorticity measured at the center of the vortex. For both angles

of attack, the maximum vorticity is recorded at 3.0c downstream with $\omega=-1.5 \frac{1}{\mathrm{~s}}$ for $\alpha=5.0^{\circ}$ and $\omega=-2.35 \frac{1}{s}$ for $\alpha=10.0^{\circ}$. Figure 3.6c shows the vortex core radius development with respect to downstream. The vortex core radius can be considered as constant even though it is getting smaller as the vortex travels downstream since the change in the radius is about $0.4 \mathrm{~mm}$ overall. Figures 3.7 show the T.K.E. distribution of the vortex before and after wandering correction at $5.0^{\circ}$ and $10.0^{\circ}$. For $\alpha=5.0^{\circ}$ and 1.0 chord length downstream the T.K.E. value is almost the same indicating that 
turbulence within the core is dominant in this region. As the vortex travels downstream, wandering corrected T.K.E. values diminishes and at 4.0 chord length downstream, the T.K.E. value is lowered to almost ambient values. For $\alpha=10.0^{\circ}$, the T.K.E. value still remains high up to 3.0 chord length downstream even after the wandering correction. And at 4.0 chord length downstream, the T.K.E. values get lower to about 0.008 within the core; however, the remaining T.K.E. values outside of the core in Figure 3.8g can be seen in the Figure 3.8h. This suggest that the peak T.K.E. value in the non re-centered data is mainly cased by wandering and the T.K.E. value on the outer side, especially the wake region, is caused by the vortex turbulence itself. Figures 3.9 show the Reynolds stress distribution of the vortex before and after wandering correction at $5.0^{\circ}$ and $10.0^{\circ}$. For $5.0^{\circ}$, the Reynolds stress distributions before and after removing the vortex wandering stay almost the same till 2.0 chord length down stream. At $c=3.0$, the Reynolds stress distribution within the core is almost neutralized after the wandering correction, indicating that the vortex roll up for $\alpha=5.0^{\circ}$ is completed. For $10.0^{\circ}$, the Reynolds stress distribution before and after removing the wandering stays almost the same till 2.0 chord length down stream. And at $c=4.0$, the Reynolds stress distribution within the core is almost neutralized after the wandering correction, indicating that the vortex roll up for $10.0 \mathrm{deg}$ is completed. 


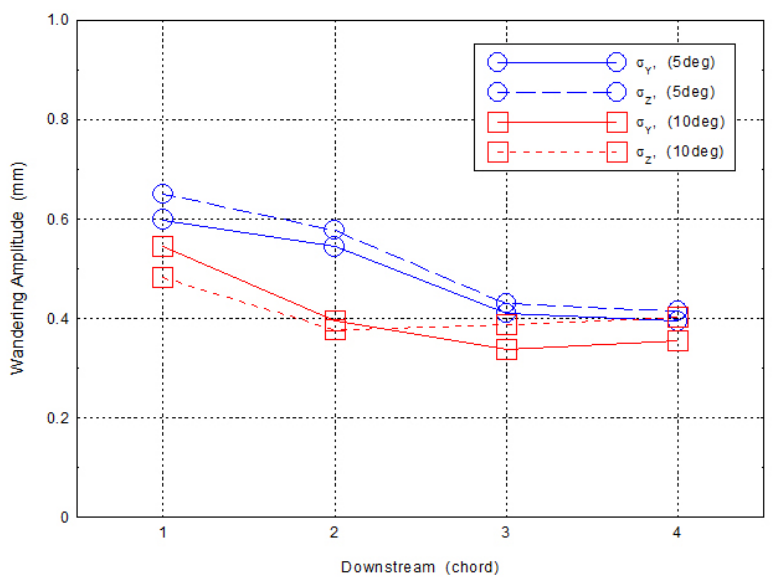

(a) Wandering amplitude.

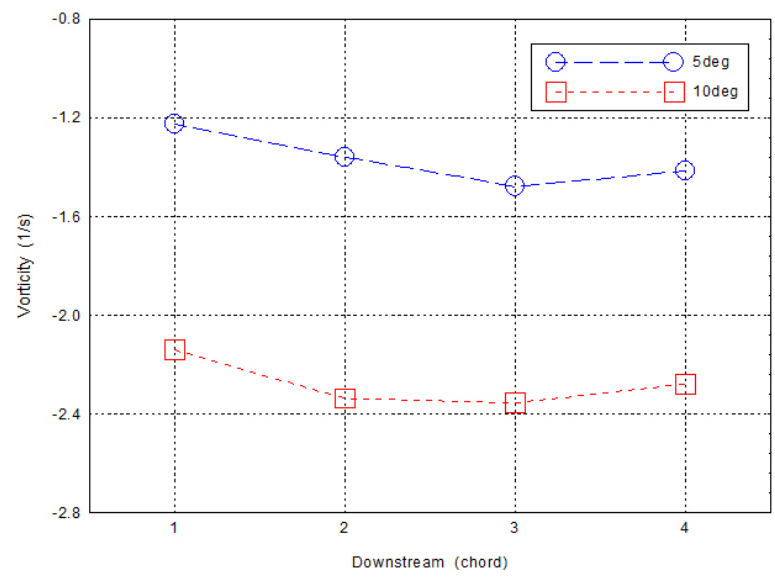

(b) Vorticity at vortex center.

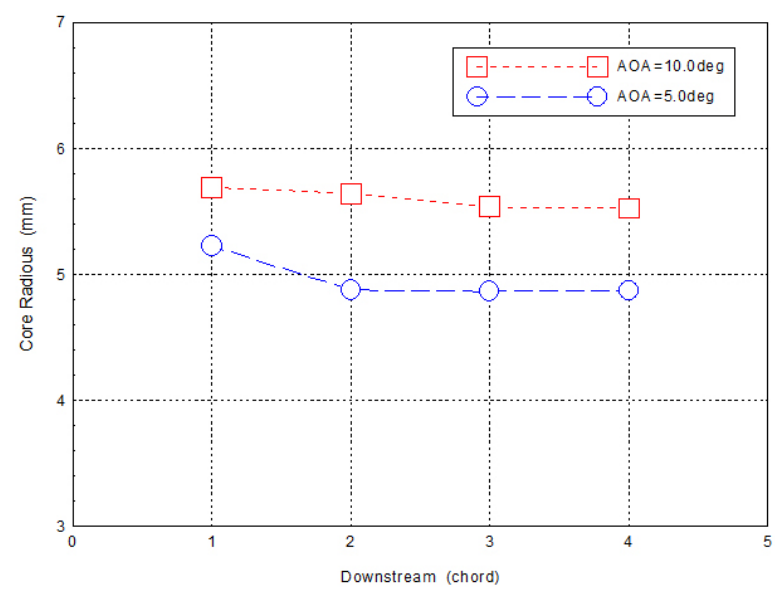

(c) Vortex core radius.

Figure 3.6: Downstream effect. 


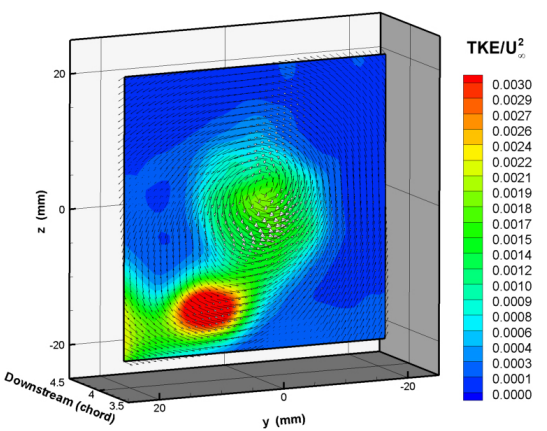

(a) 1.0 chord length downstream

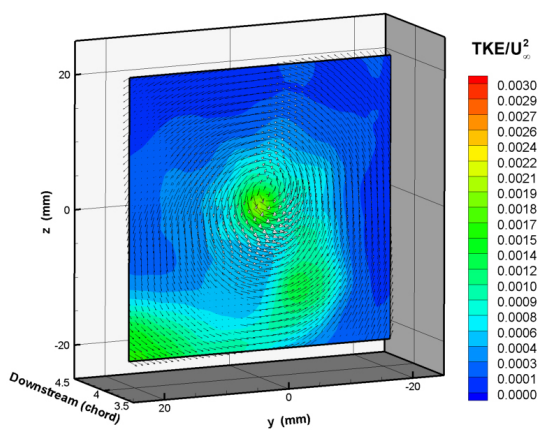

(c) 2.0 chord length downstream

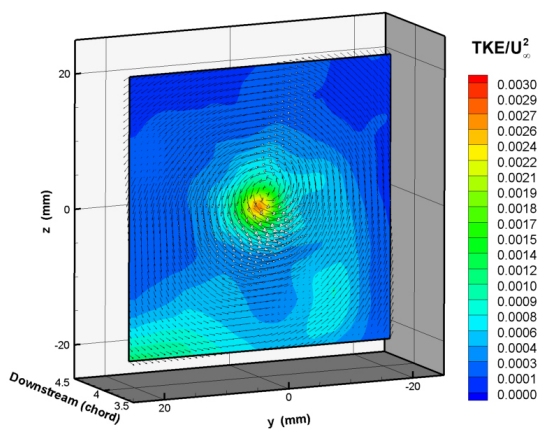

(e) 3.0 chord length downstream

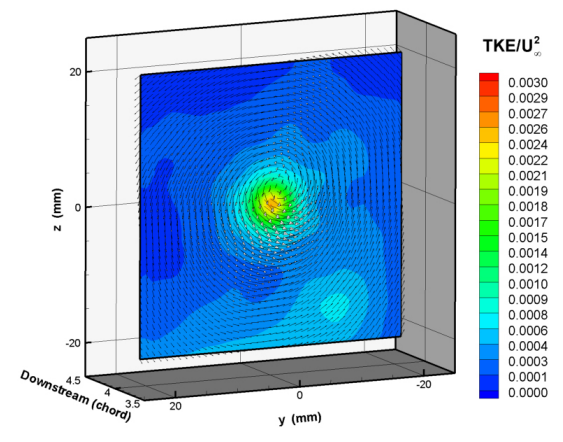

(g) 4.0 chord length downstream

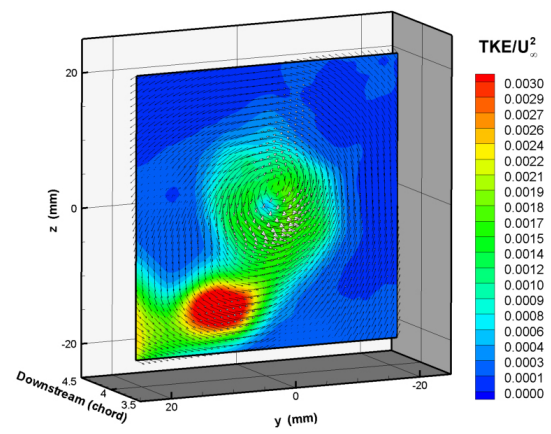

(b) 1.0 chord length, re-centered

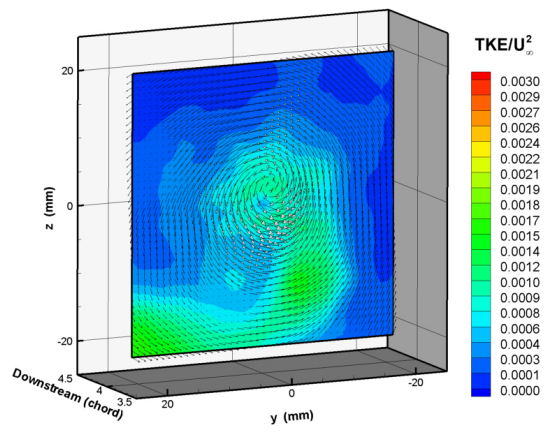

(d) 2.0 chord length, re-centered

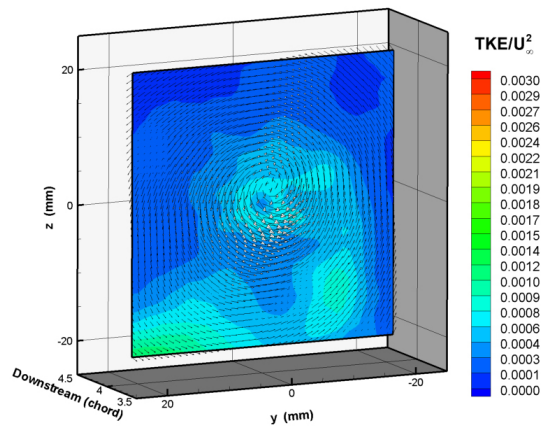

(f) 3.0 chord length, re-centered

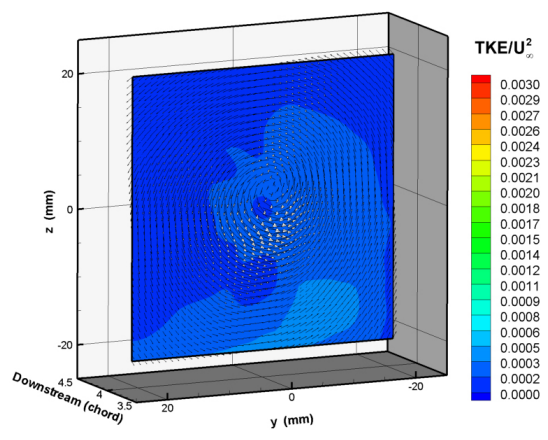

(h) 4.0 chord length, re-centered

Figure 3.7: Normalized T.K.E. distributions from 1.0 to 4.0 chord length downstream at $\alpha=5.0 \mathrm{deg}$. 


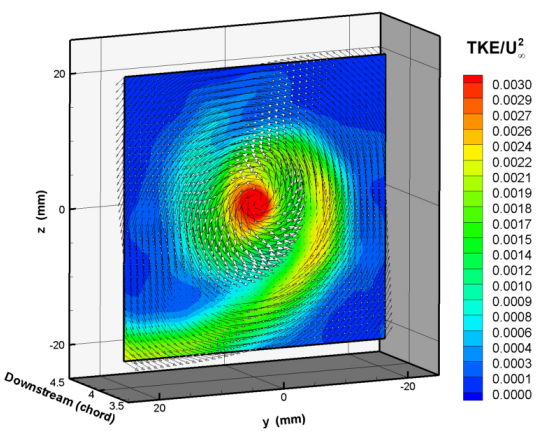

(a) 1.0 chord length downstream

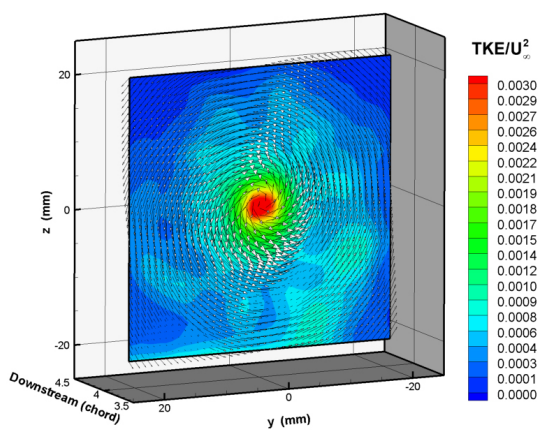

(c) 2.0 chord length downstream

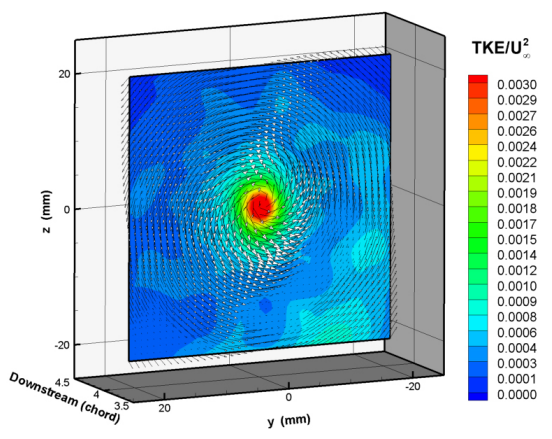

(e) 3.0 chord length downstream

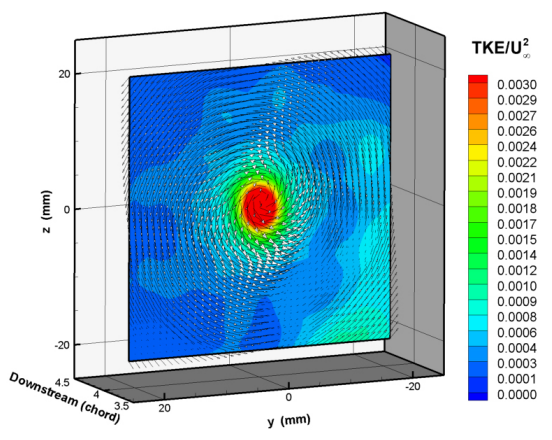

(g) 4.0 chord length downstream

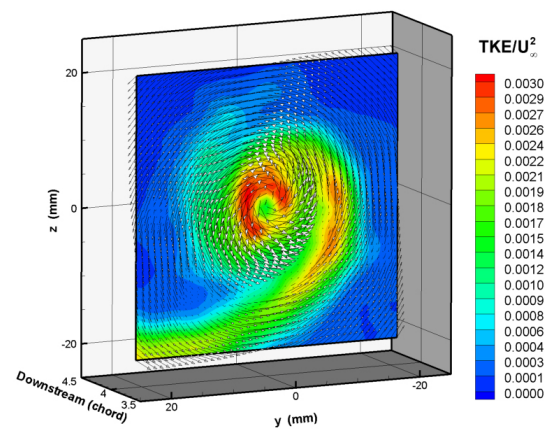

(b) 1.0 chord length, re-centered

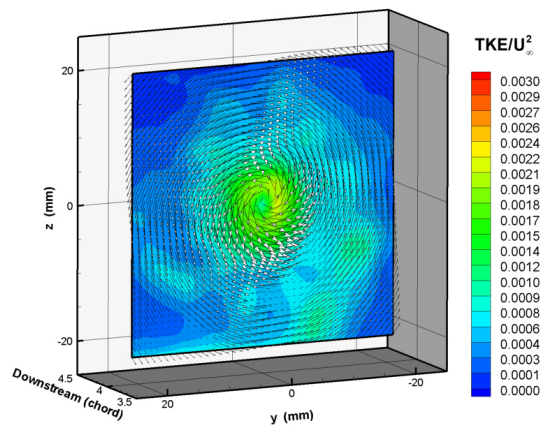

(d) 2.0 chord length, re-centered

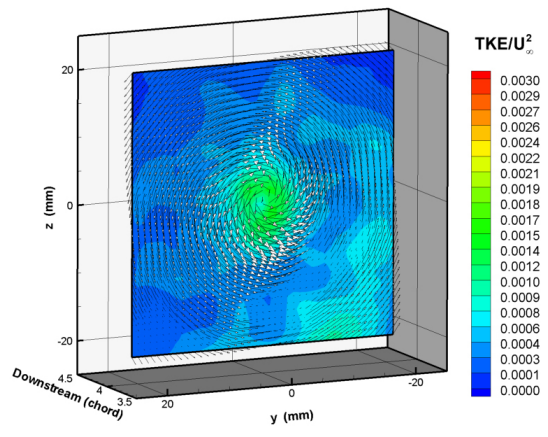

(f) 3.0 chord length, re-centered

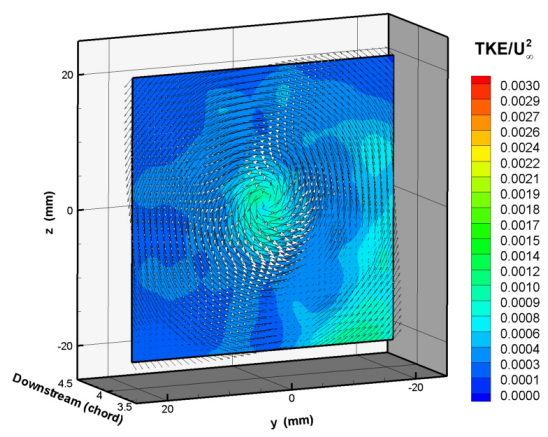

(h) 4.0 chord length, re-centered

Figure 3.8: Normalized T.K.E. distributions for 1.0 and 2.0 chord length downstream at $\alpha=10.0$ deg. 


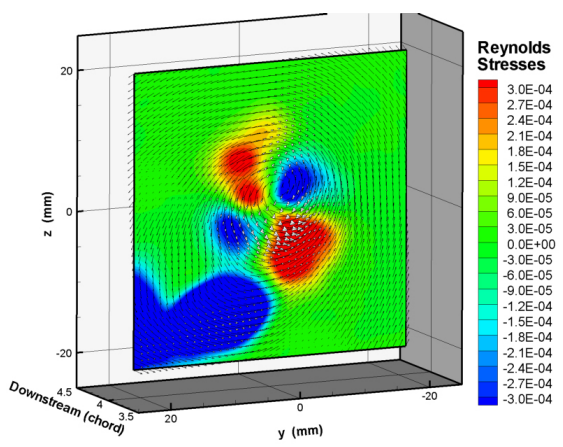

(a) 1.0 chord length downstream

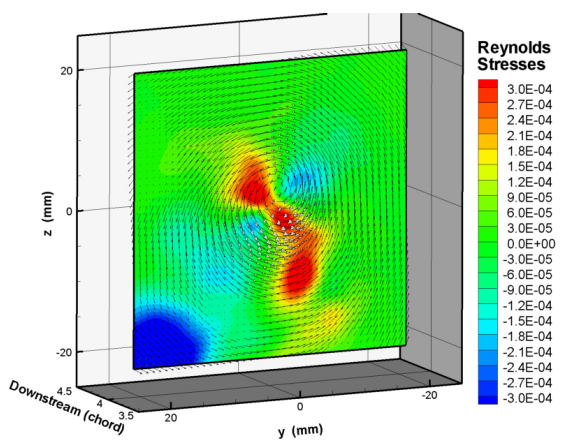

(c) 2.0 chord length downstream

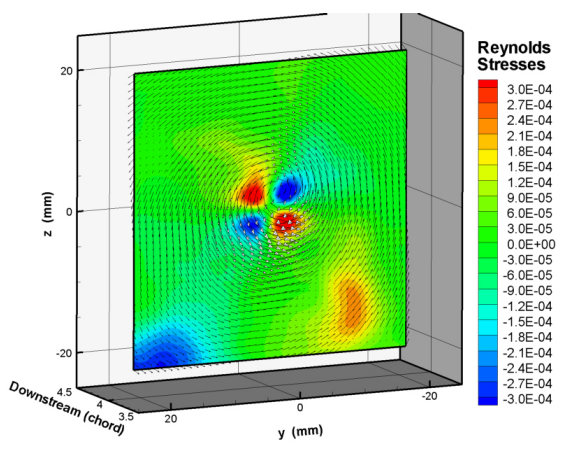

(e) 3.0 chord length downstream

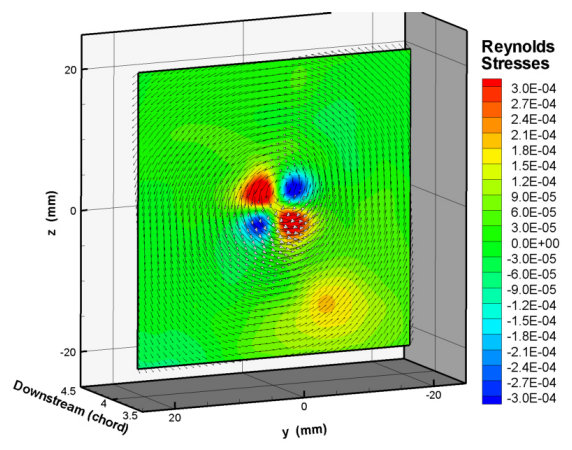

(g) 4.0 chord length downstream

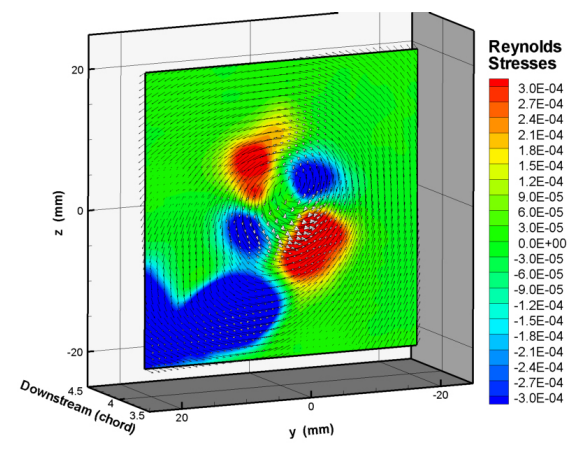

(b) 1.0 chord length, re-centered

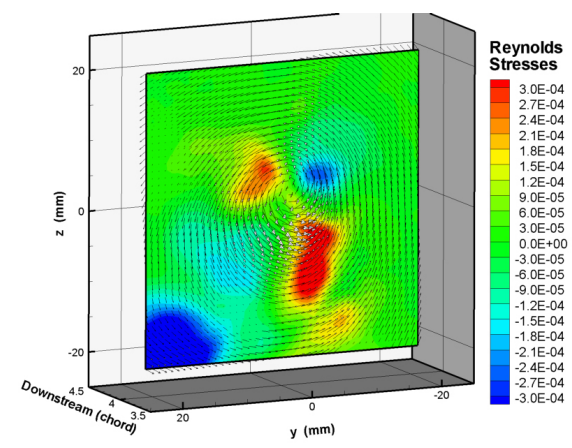

(d) 2.0 chord length, re-centered

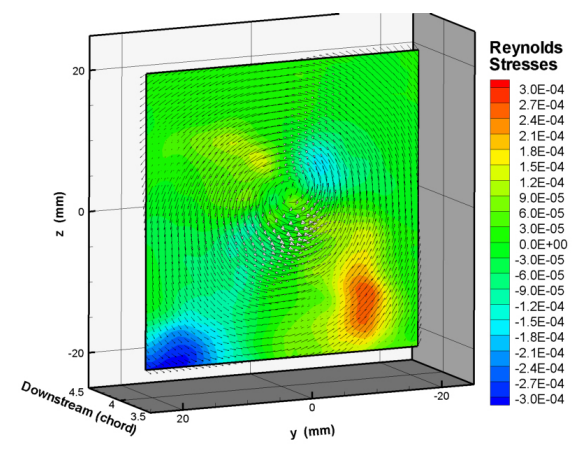

(f) 3.0 chord length, re-centered

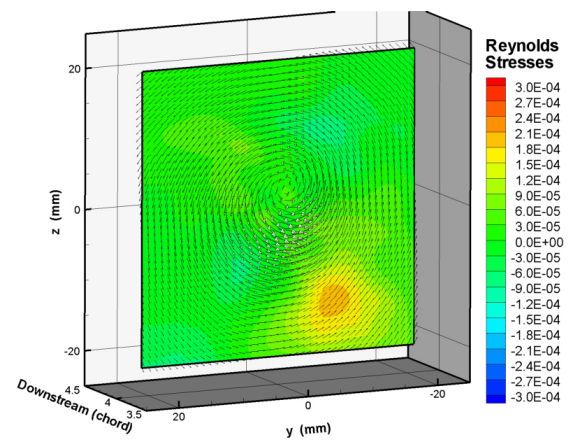

(h) 4.0 chord length, re-centered

Figure 3.9: Normalized Reynolds Stress distributions from 1.0 to 4.0 chord length downstream at $\alpha=5.0 \mathrm{deg}$. 


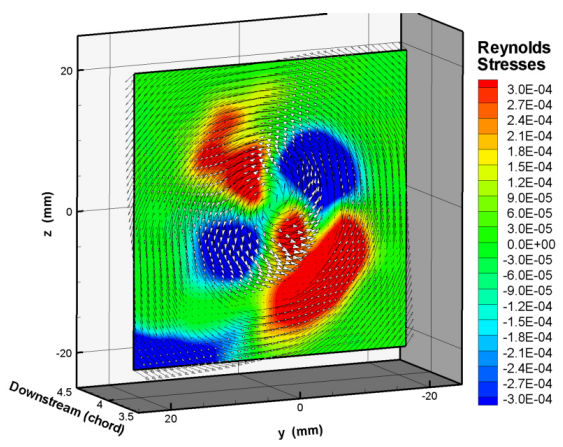

(a) 1.0 chord length downstream

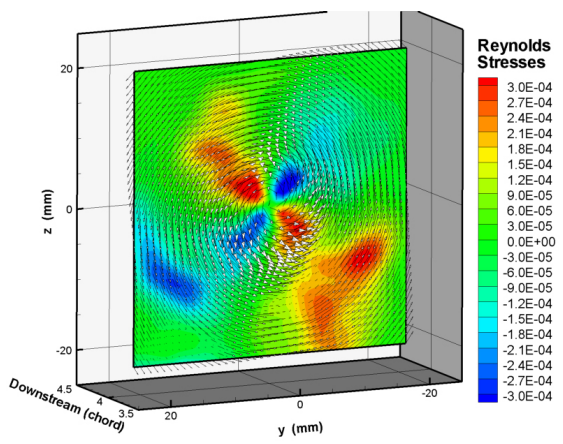

(c) 2.0 chord length downstream

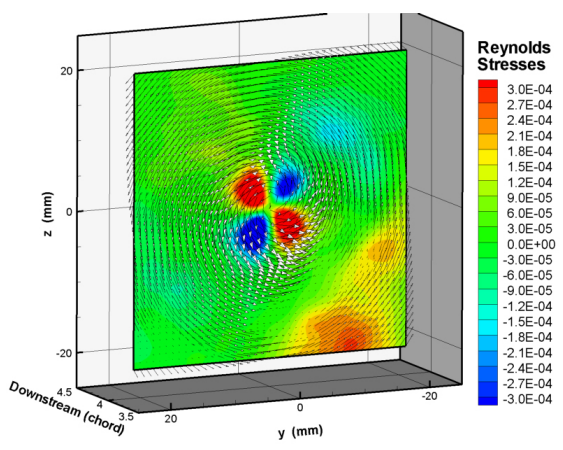

(e) 3.0 chord length downstream

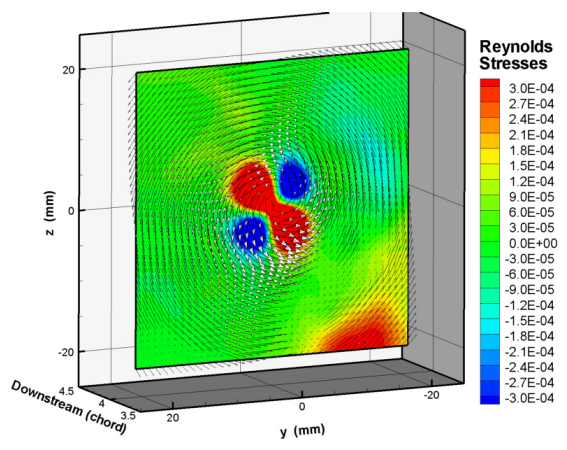

(g) 4.0 chord length downstream

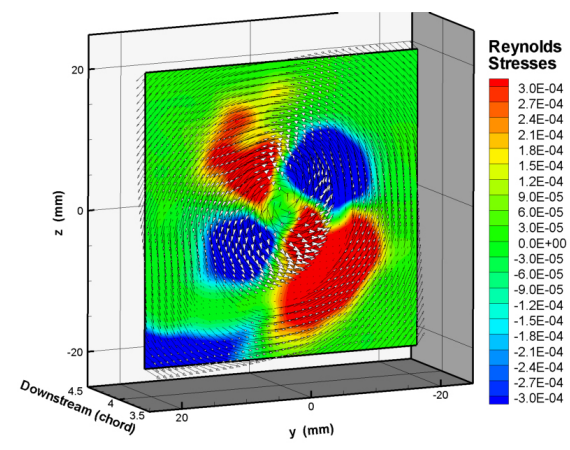

(b) 1.0 chord length, re-centered

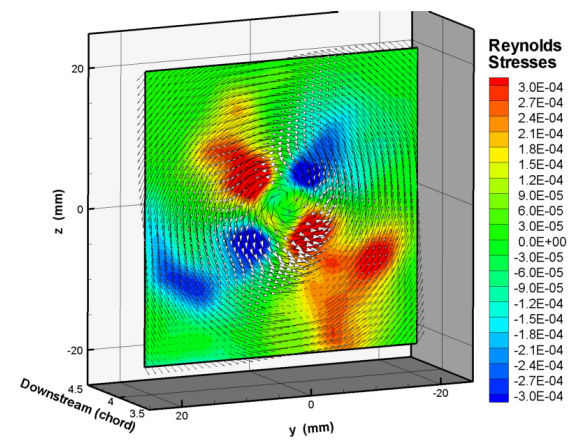

(d) 2.0 chord length, re-centered

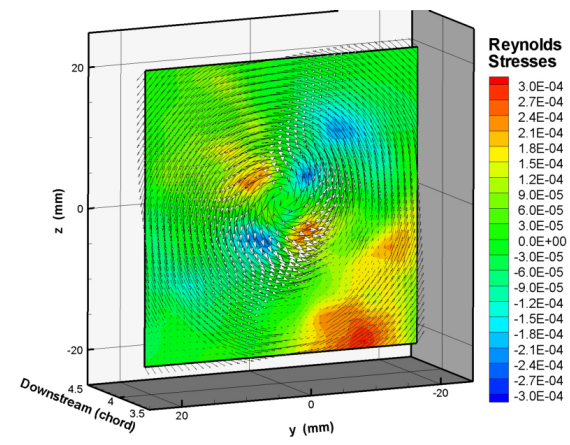

(f) 3.0 chord length, re-centered

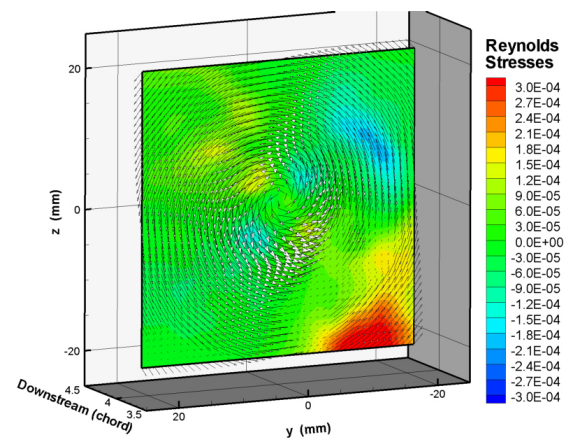

(h) 4.0 chord length, re-centered

Figure 3.10: Normalized Reynolds Stress distributions from 1.0 to 4.0 chord length downstream at $\alpha=10.0 \mathrm{deg}$. 


\subsection{Devenport Method}

This section presents the results of the Devenport's method described in section 1.2.

For this investigation, the Devenport method was applied to two different angles of attack, $\alpha=2.0^{\circ}$ and $\alpha=4.0^{\circ}$. Figure 3.11a shows the tangential velocity profiles of the vortex along the positive z-axis at $\alpha=2.0^{\circ}$ and Figure 3.11b shows the tangential velocity profile with the same flow conditions with $\alpha=4.0^{\circ}$. For both figures, the blue solid curve represents the non re-centered velocity profile, i.e., velocity profile with vortex wandering. The black solid curve represents the SPIV re-centered velocity profile i.e., instantaneously corrected wandering free velocity profile and red symbols represent the Devenport method re-centered velocity profile, i.e., numerically derived wandering free velocity profile. At $\alpha=2.0^{\circ}$, as shown in Figure 3.11a, the SPIV method and Devenport method agree very well with each other from the vortex core center to the core radius. After passing the core radius, the Devenport correction curve starts to diverge from the SPIV curve and converging to the non re-centered curve. This is because the equations used to derive the Devenport curve are formulated to converge to the non re-centered profile at the outer region of the vortex. For this case, the SPIV corrected curve is more like the off-set profile from the non re-centered profile, so that there will be some discrepancy between those two curves at the outer region of the vortex. Figure 3.11b shows the velocity profile at $\alpha=4.0^{\circ}$. For this case, the SPIV corrected profile and Devenport corrected profile within the vortex core do not match to each other, or the Deveport method recovered less tangential velocity than SPIV method. After passing the vortex radius, all three velocity profiles converged to each other indicating that there is not much wandering effect occurring at this particular outer region of the vortex core. By observing the profiles on Figures 3.11a and 3.11b, the Devenport's method is not consistently predicting the correct velocity profile in radial direction. For the $\alpha=2.0^{\circ}$ case, they have a good agreement within the core but for $\alpha=4.0^{\circ}$ case they 
Table 3.1: Wandering amplitude data for SPIV method and Devenport method

\begin{tabular}{cccccccc} 
& \multicolumn{3}{c}{ SPIV Method } & & \multicolumn{3}{c}{ Devenport Method } \\
\cline { 2 - 4 } \cline { 5 - 7 }$\alpha(\mathrm{deg})$ & $\sigma_{y}(\mathrm{~mm})$ & $\sigma_{z}(\mathrm{~mm})$ & $\rho$ & & $\sigma_{y}(\mathrm{~mm})$ & $\sigma_{z}(\mathrm{~mm})$ & $\rho$ \\
\hline 2.0 & 0.96 & 0.94 & -0.089 & & 0.79 & 0.94 & -0.056 \\
4.0 & 0.45 & 0.47 & -0.004 & & 0.44 & 0.50 & -0.061
\end{tabular}

have a good agreement at the outer core region. This inconsistency happens because the Devemport method uses only one velocity profile on the positive y axis. This is not sufficient to fully recover the wandering free velocity profile accurately. One of the assumptions made by Devenport et al. (1996) was to assume a vortex axisymmetric, but the real wing-tip vortex is not perfectly axisymmetric and has the distinctive profile shape depending on the profile extracting locations. Therefore Devenport method is a good way to approximate the wandering-free velocity profile, but not sufficient to predict the accurate profile.

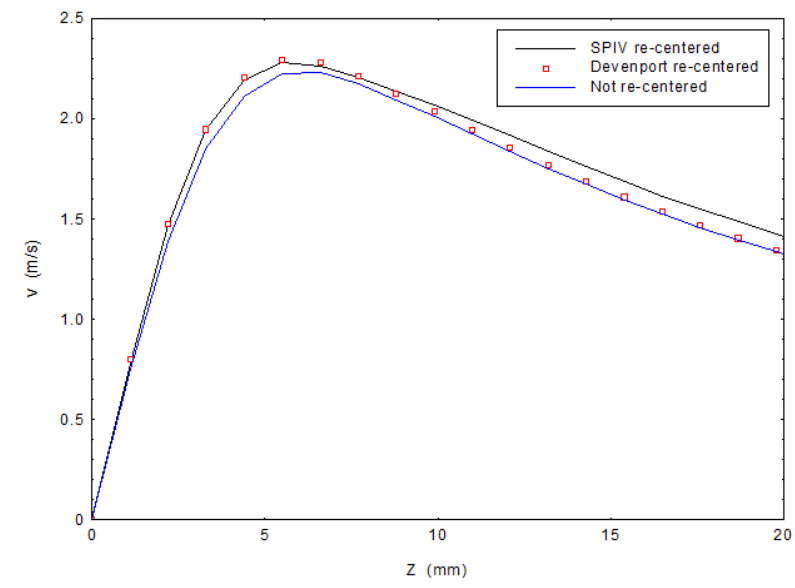

(a) $\alpha=2.0^{\circ}$

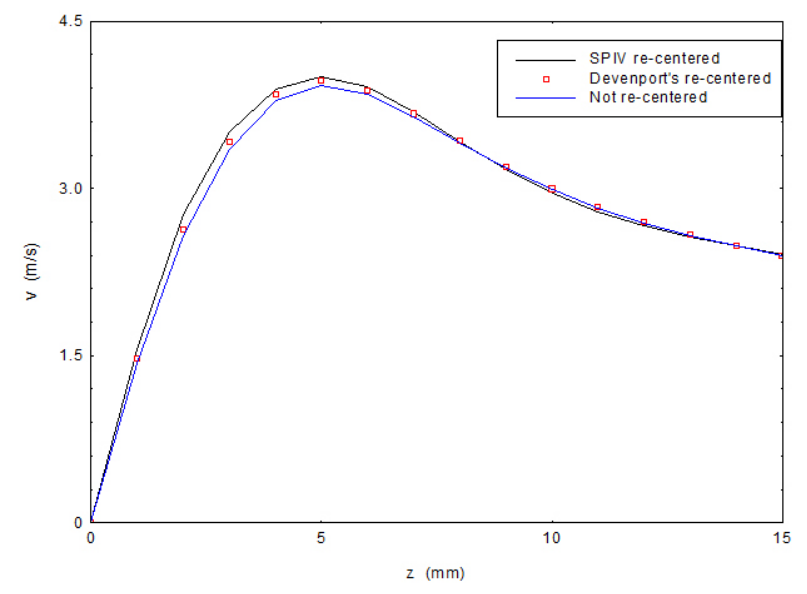

(b) $\alpha=4.0^{\circ}$

Figure 3.11: Re-centered tangential velocity profiles by SPIV and Devenport methods. 


\subsection{Wall Effect}

As described in the Section 2.3.1, the additional wall is inserted in the wind tunnel test section to change the wall-to-tip distance from 6.0in to 4.0, 2.0, and 1.0in. This section presents the effect of these additional inner walls on the vortex structures and behavior in the wind tunnel.

Figures $3.12 \mathrm{a}$ and $3.12 \mathrm{~b}$ show the projection of the vortex centers and the average vortex center with different wall lengths. When there is no additional wall, i.e., 6.0in wall-to-tip distance, the vortex center lies around $z=-5.2$, which is near the trailing edge height. Then as the wall gets closer to the wing tip, the vortex starts moving upward.

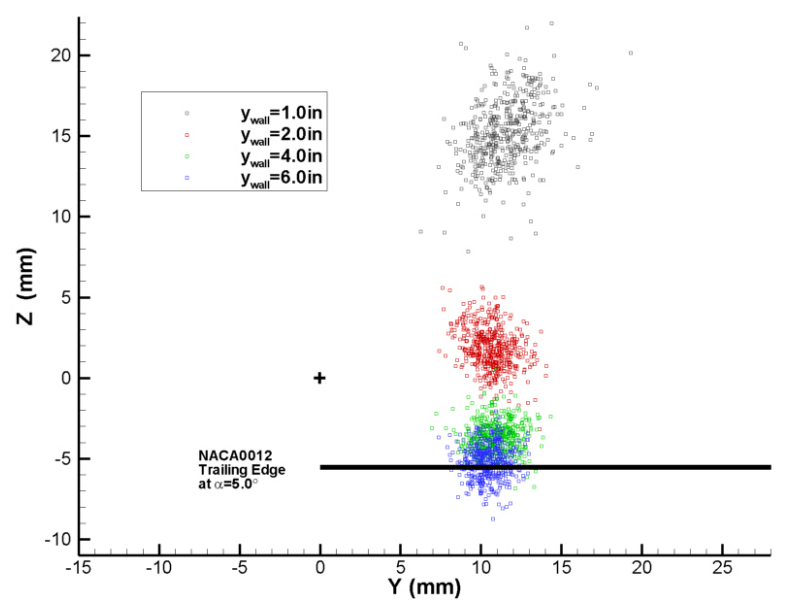

(a) Vortex scattering with additional wall

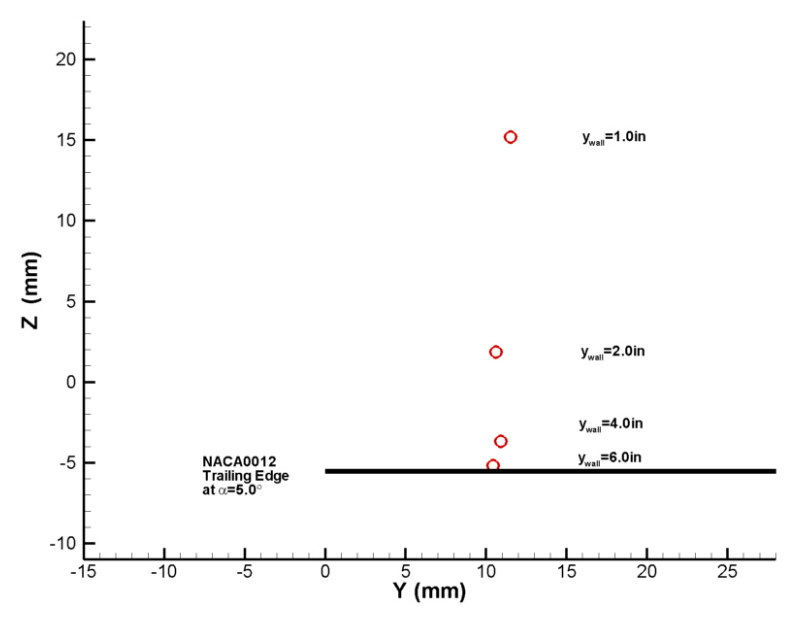

(b) Average vortex center with additional wall

Figure 3.12: Effect of upwash by the additional walls.

When the wall exists, it acts as the mirror vortex image which exists on the other side of the wall. Therefore there will be a stronger influence of the vortex upwash instead of a down wash, which drives the vortex upward. The correlation between the wall to the wing-tip distance and the vortex up wash driven height can be shown in log-log scale plot as in Figure3.13. By fitting the curve with the power equation, the equation 
is determined as $\ln y=-1.69 \log x+8.61$. These coefficients may vary with the flow condition and wing angle of attack, as the equation is depending on the vortex radius.

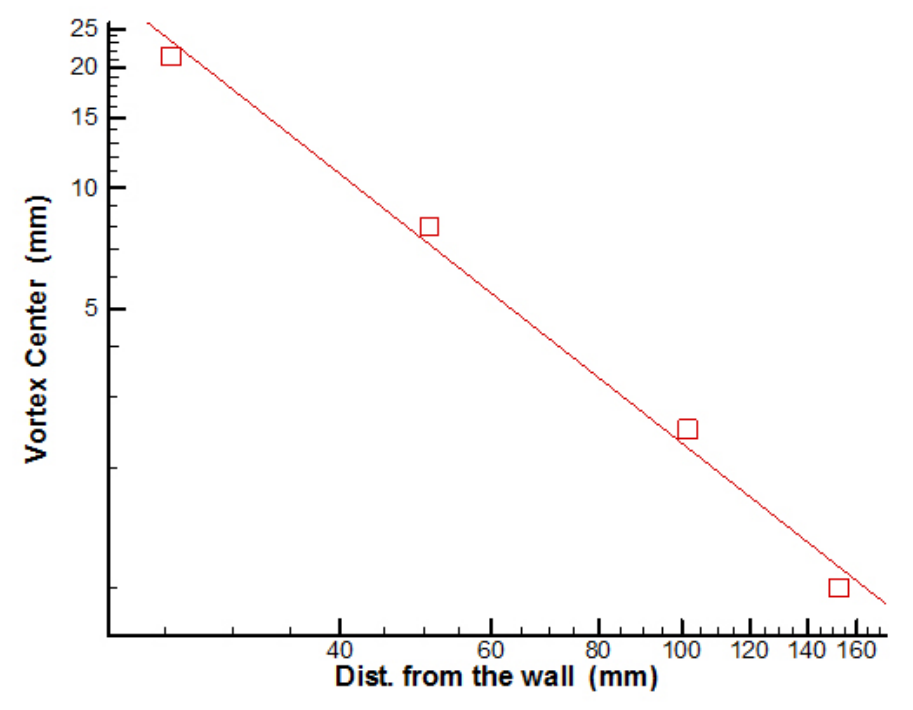

Figure 3.13: Correlation of wall-to-tip distance and vortex upward travel.

The Figures 3.14 and 3.15 show the turbulence and Reynolds stresses distribution around the vortex affected by the additional inner walls. Figure 3.14 shows the T.K.E. distribution in and vicinity of the vortex and the left column figures show the T.K.E. distribution before correcting the wandering effect and the right column figures show the T.K.E. distribution without the wandering. As the wall gets closer from 4.0in to 1.0in, the T.K.E. values get higher; especially, there is a significant increase in T.K.E. values from 2.0in to 4.0in. When wall gets closer by 2 in from Figures 3.14a to 3.14c, the total T.K.E. distribution with the vortex wandering is about the same or a little bit increase in the core turbulence size. After removing the wandering effect, which is shown in Figures $3.14 \mathrm{~b}$ and $3.14 \mathrm{~d}$, it is clearly shown that moving the inner wall closer to the wing-tip will increase the turbulence level within the vortex core more than twice. When the wall is further pushed up to the 1.0in tip-to-wall distance, the both non re-centered and re-centered distribution (shown in Figure 3.14e and 3.14f) look the same. These figures 
suggest that T.K.E., at 1.0in distance, is dominated by the turbulence within the vortex itself and the turbulence by the wandering has little or no effect impact on the total T.k.E. distributions. Table 3.2 shows the wandering statistics of the wing-tip vortex with the wall effect. The introduction of the additional wall increased the wandering amplitude about twice from $\sigma \approx 0.4$ to $\approx 0.7$, and the center location distribution is less axisymmetric, e.g., $\sigma_{y}=0.72$ and $\sigma_{z}=0.94$ with $2.0 \mathrm{in}$ wall-to-tip distance. For the 1.0in tip-to-wall distance case, the vortex wanders $50 \%$ more in the y-axis direction. The vortex radius tends to get larger from $4.9 \mathrm{~mm}$ to $6.33 \mathrm{~mm}$ as the wall get closer from thirty to less than ten radii away from the tip; however, at 1.0in wall-to-tip, the radius gets smaller because the physical wall is getting closer to less than five vortex radii away. While the vortex behaves differently with the additional walls, the vorticity is not affected by the wall effect and stays around $\omega \approx-3.11 / \mathrm{s}$ at up to $8.0 r_{c}$. When the wall is moved closer to less than 5 radii away from the tip, the vorticity gets smaller by $10 \%$. Observing from the vortex structure and behavior, the vortex seems to interact with the mirror image vortex on the other side of the wall up to $8.0 r_{c}$ away; however, if the wall gets to within 5.0 or 4.0 radii way from the wing-tip, vortex would have interaction with the physical wall more and behaves more in a chaotic fashion. 


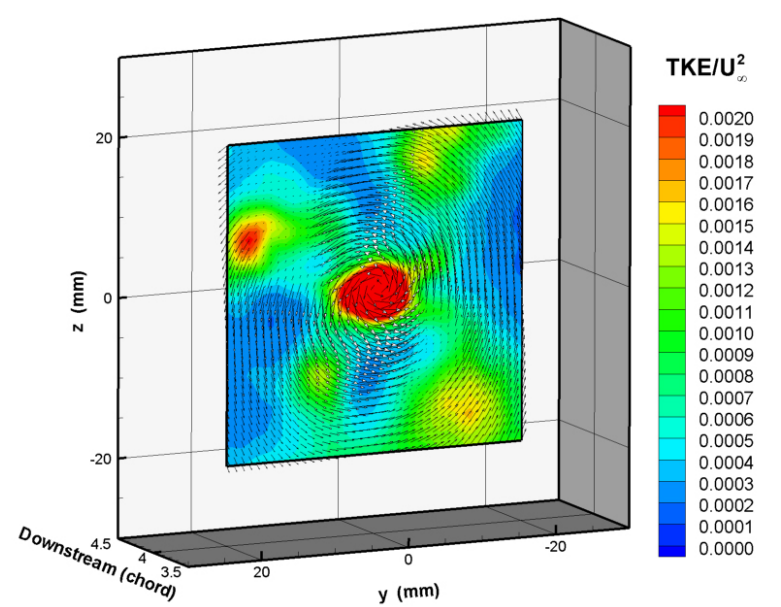

(a) 4.0in tip to wall

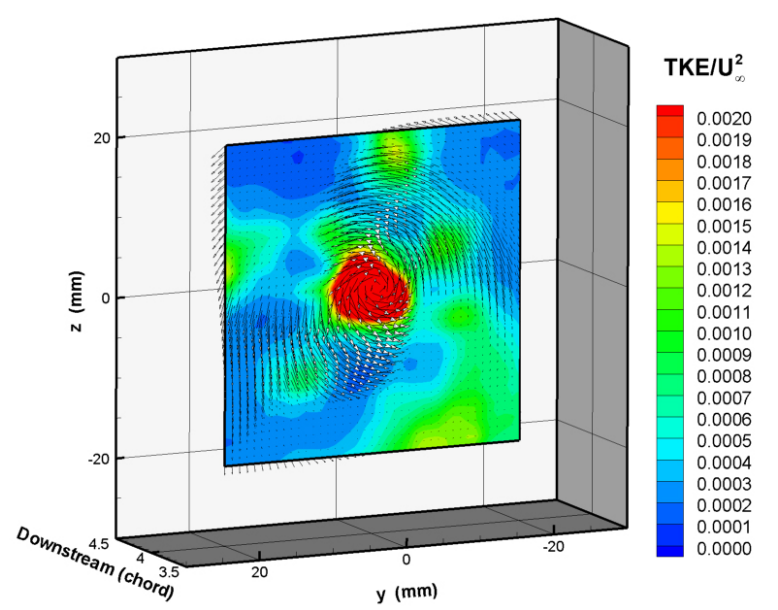

(c) 2.0in tip to wall

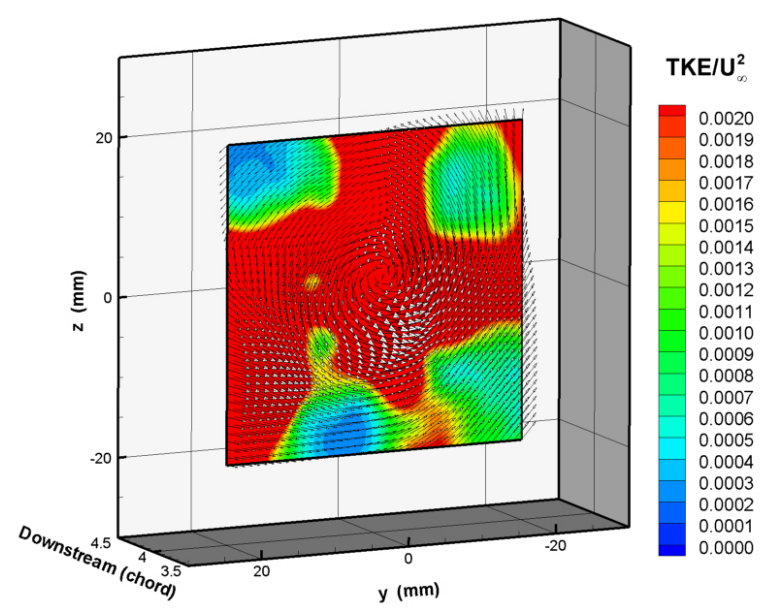

(e) 1.0in tip to wall

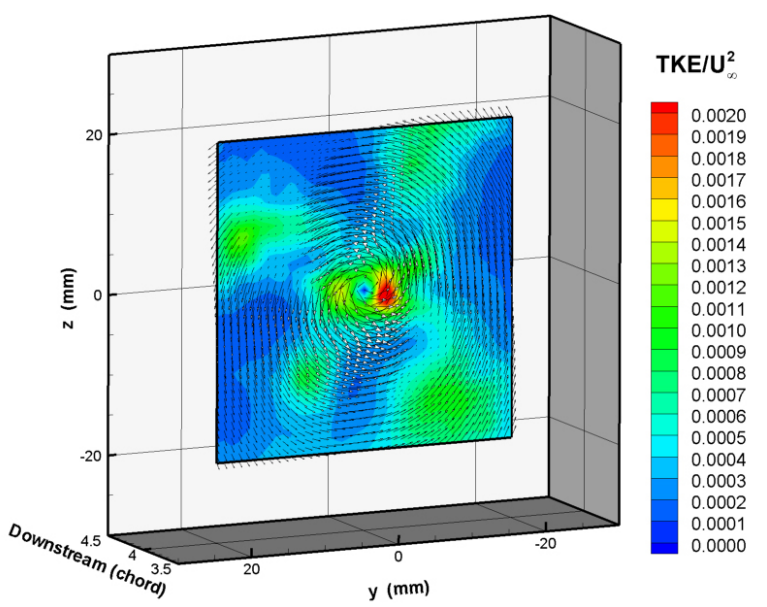

(b) 4.0in tip to wall, re-centered

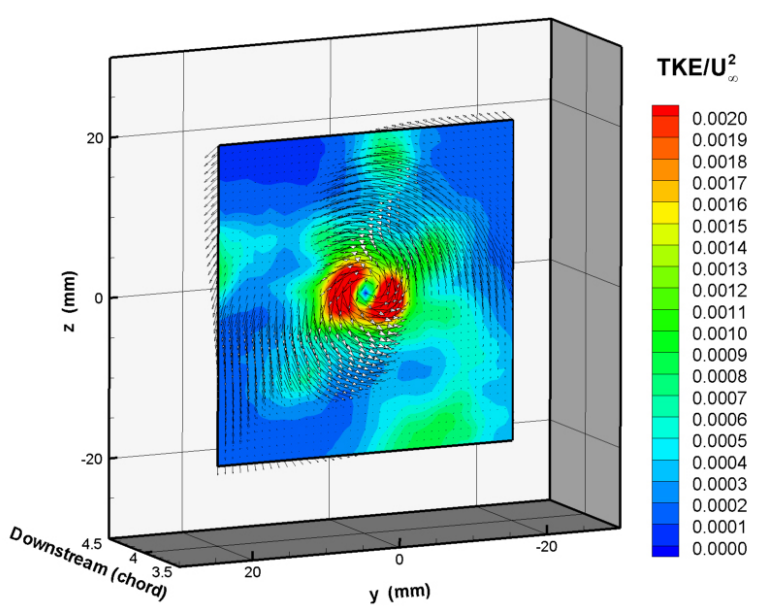

(d) 2.0in tip to wall, re-centered

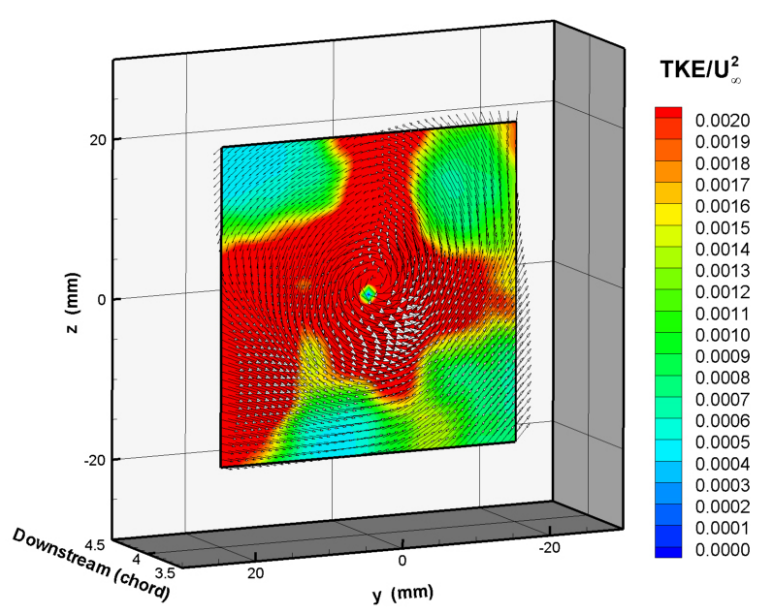

(f) 1.0in tip to wall, re-centered

Figure 3.14: T.K.E. distributions for wall effect. 


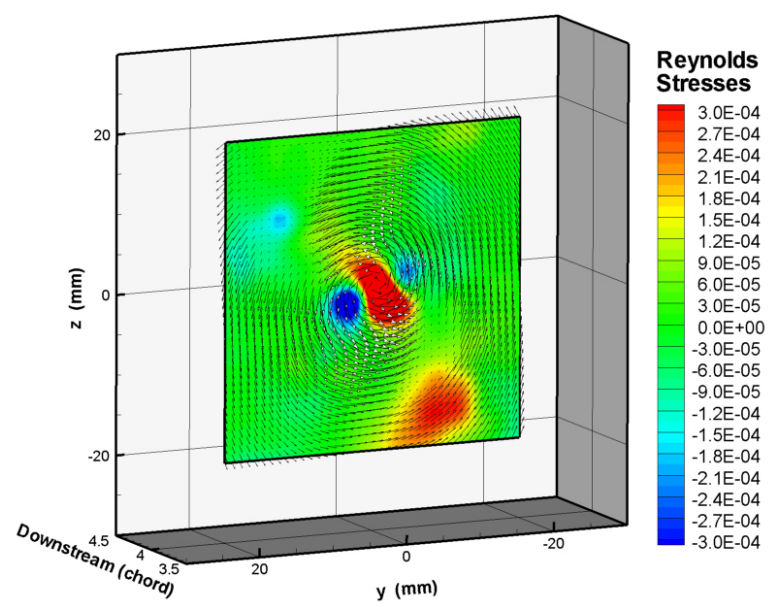

(a) 2in wall

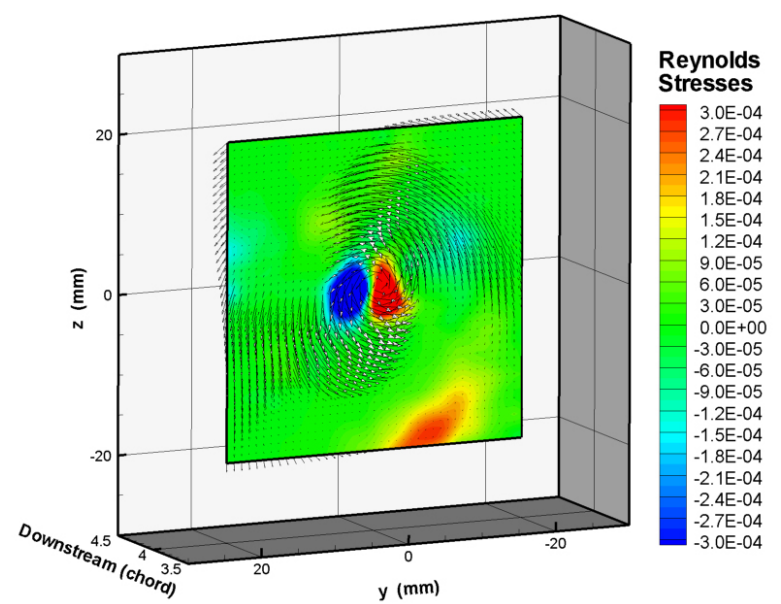

(c) 4in wall

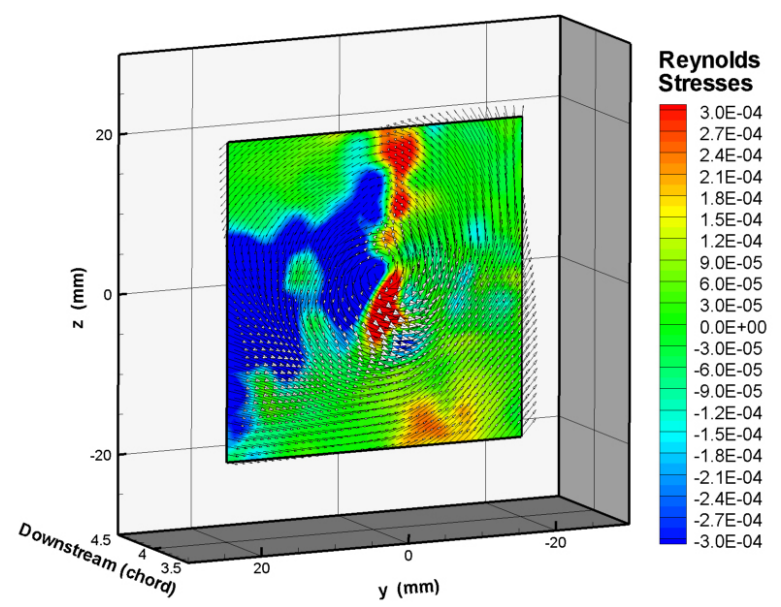

(e) 5in wall

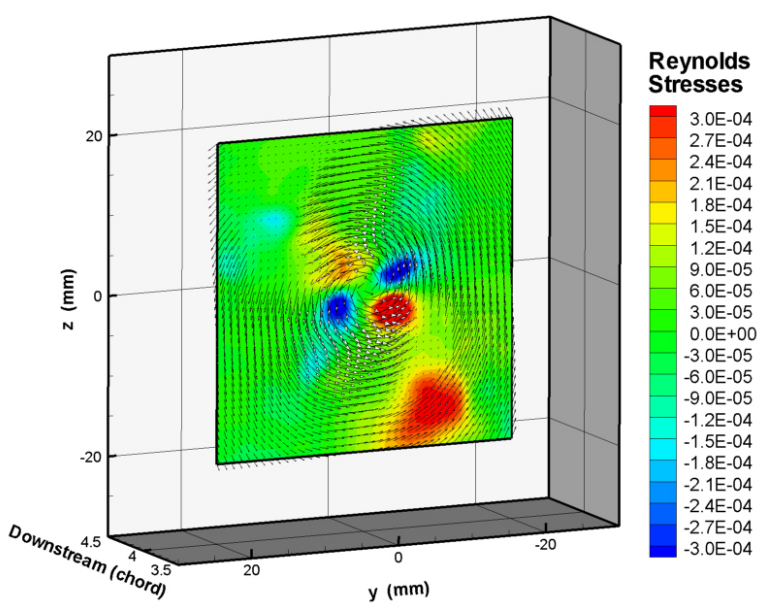

(b) 2in wall, re-centered

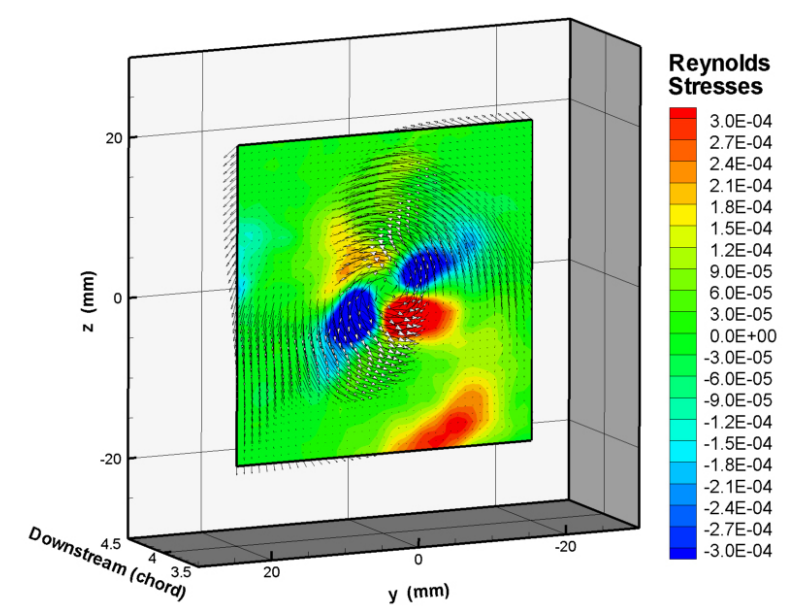

(d) 4in wall, re-centered

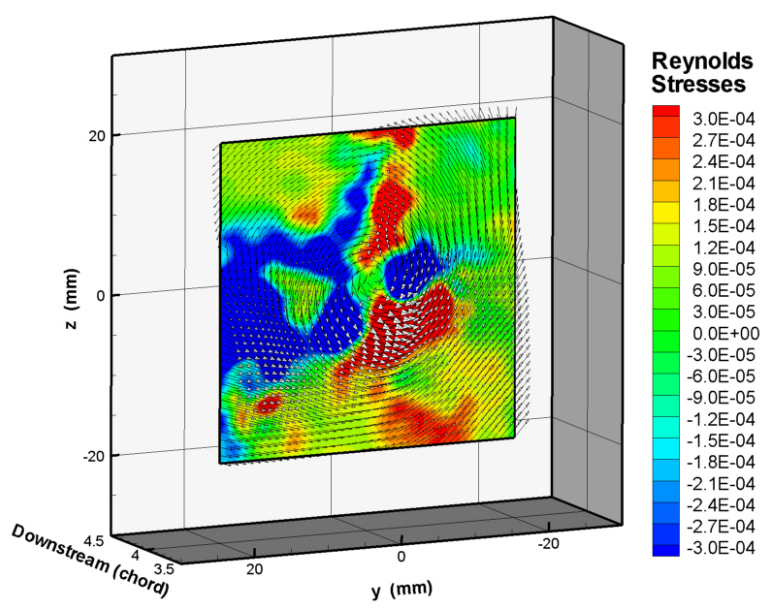

(f) 5in wall, re-centered

Figure 3.15: Normalized Reynolds Stress distributions for wall effect. 
Table 3.2: Numerical data for SPIV method

\begin{tabular}{|c|c|c|c|c|c|c|c|c|c|}
\hline \multirow[b]{2}{*}{$\begin{array}{c}\alpha \\
(\operatorname{deg})\end{array}$} & \multirow[b]{2}{*}{$R e$} & \multirow[b]{2}{*}{$x / c$} & \multirow[b]{2}{*}{$\begin{array}{c}\text { Tip-to-Wall } \\
\text { (in) }\end{array}$} & \multicolumn{5}{|c|}{ SPIV Method } & \multirow[b]{2}{*}{$\begin{array}{c}\omega \\
(1 / s)\end{array}$} \\
\hline & & & & $\begin{array}{c}\sigma_{y} \\
(\mathrm{~mm})\end{array}$ & $\begin{array}{c}\sigma_{z} \\
(\mathrm{~mm})\end{array}$ & $\rho$ & $\begin{array}{c}r_{c} \\
(\mathrm{~mm})\end{array}$ & $\begin{array}{c}v_{\theta} \\
(\mathrm{m} / \mathrm{s})\end{array}$ & \\
\hline 2.0 & $1.15 \times 10^{5}$ & 4.0 & $6.0\left(28.9 r_{c}\right)$ & 0.96 & 0.94 & -0.089 & 5.37 & 2.00 & -1.20 \\
\hline 4.0 & $1.15 \times 10^{5}$ & 4.0 & $6.0\left(31.5 r_{c}\right)$ & 0.45 & 0.47 & -0.004 & 4.85 & 3.63 & -2.66 \\
\hline 6.0 & $1.15 \times 10^{5}$ & 4.0 & $6.0\left(30.8 r_{c}\right)$ & 0.34 & 0.36 & -0.011 & 5.00 & 5.05 & -3.64 \\
\hline 8.0 & $1.15 \times 10^{5}$ & 4.0 & $6.0\left(26.6 r_{c}\right)$ & 0.32 & 0.33 & -0.093 & 5.65 & 6.10 & -3.65 \\
\hline 10.0 & $1.15 \times 10^{5}$ & 4.0 & $6.0\left(24.9 r_{c}\right)$ & 0.35 & 0.37 & 0.059 & 5.82 & 7.43 & -4.36 \\
\hline 12.0 & $1.15 \times 10^{5}$ & 4.0 & $6.0\left(24.1 r_{c}\right)$ & 0.33 & 0.37 & -0.176 & 6.19 & 8.60 & -4.78 \\
\hline 5.0 & $1.15 \times 10^{5}$ & 4.0 & $4.0\left(18.9 r_{c}\right)$ & 0.74 & 0.81 & -0.053 & 5.37 & - & -3.17 \\
\hline 5.0 & $1.15 \times 10^{5}$ & 4.0 & $2.0\left(8.0 r_{c}\right)$ & 0.72 & 0.94 & 0.155 & 6.33 & - & -3.12 \\
\hline 5.0 & $1.15 \times 10^{5}$ & 4.0 & $1.0\left(4.5 r_{c}\right)$ & 1.09 & 1.59 & 0.276 & 5.67 & - & -2.78 \\
\hline
\end{tabular}




\section{CHAPTER 4. CONCLUSION}

In the present study, a high-resolution Stereoscopic Particle Image Velocimetry (SPIV) system, which is capable of achieving quantitative measurements of all three components of instantaneous flow velocity vectors, was used to conduct detailed flow field measurements to quantify the transient behavior of the wing-tip vortex generated from a rectangular NACA0012 airfoil.

From this study, any correlation between the vorticity and the vortex wandering cannot be seen on certain angles of attack. Up to $\alpha=6.0^{\circ}$, the vortex wandering becomes smaller as the vorticity increases, but after $\alpha=8.0^{\circ}$, the wandering stays the same as the vorticity increases by $50 \%$. The same conclusion can be drawn for the vortex radius as well. The radius gets smaller between $\alpha=2.0^{\circ}$ and $\alpha=4.0^{\circ}$, but after $\alpha=4.0^{\circ}$, the radius size increases by $30 \%$. By comparing the re-centered and non recentered contour plots, it can be said that the most of the higher T.K.E. and Reynolds stresses in the vortex core are coming from the vortex wandering for 4.0c downstream.

From the initial roll up to 4.0 chord length down stream, the vortex core radius is constant. To be precise, the radius actually gets smaller, but the difference was $0.4 \mathrm{~mm}$

which is not significant compare to the vortex radius. Right after the trailing edge of the wing, the re-centered T.K.E. and Reynolds stress distributions are about the same as before the wandering correction which is indicating that the tip vortex is highly turbulent near 1.0c and 2.0c downstream. The Reynolds stress distributions indicate that the vortex rollup is completed after 3.0c downstream for $\alpha=5.0^{\circ}$ and $4.0 \mathrm{c}$ for $\alpha=10.0^{\circ}$ since the stresses are almost neutralized after the center correction. 
The Devenport's method under and/or over predicts the tangential velocity depending on the radial distance from the center and the prediction is not consistently accurate. This is because the calculation is depending on only one tangential velocity profile. The author found out that this is not sufficient to fully recover the accurate wandering free velocity profile. Therefore, the Devenport's method should only be limited to approximate the velocity profile and with reasonably larger wandering amplitude, perhaps $\sigma>r_{c} / 5$, for satisfactory results since the smaller wandering amplitude means less smearing of the velocity by the wandering. If the accurate wandering free velocity profile is necessary, then the spatially resolved instantaneous non-intrusive technique like PIV or SPIV is desired.

When the wall gets closer to the wing-tip vortex, it will increase the T.K.E. levels. When the wall is set further apart the T.K.E. comes from the wandering and the vortex turbulence itself, but when the wall gets closer enough to about $4.5 r_{c}$, then the T.K.E. is solely from the turbulence level of the vortex itself and there is a little or no impact from the wandering. As the wall get closer to the wing tip, the vortex wanders more and the vortex radius gets bigger hence the image vortex on the other side of the wall effect is big. But as the wall gets even closer to within the four or five vortex radii away, the vortex starts to wander in z-direction more but the radius and vorticity get smaller. Since the vortex starts reacting with a physical wall more and there are sudden reduction in the radius size and vorticity, this region might be considered as the transitional region between the $3 \mathrm{D}$ flow to $2 \mathrm{D}$ flow over the wing. 


\section{BIBLIOGRAPHY}

Adrian, R. J. (1991). Particle-imaging techniques for experimental fluid mechanics. Ann. Rev. of Fluid Mech., 23, 261-304.

Bailey, S. C. C. and Tavoularis, S. (2008). Measurements of the velocity field of a wing-tip vortex, wandering in grid turbulence. J. Fluid Mech., 601, 281-315.

Birch, D., Lee, T., Mokhtarian, F., and Kafyeke, F. (2004). The structure and induced drag of a tip vortex. J. Aircraft, Vol. 41, No. 5, 1138-1145.

Cerreteli, C. and Williamson, C. H. K. (2003). The physical mechanism for vortex merging. J. of Fluid Mech., Vol. 475, 41-77.

Chow, J. S., Zilliac, G. G., and Bradshaws, P. (1997). Mean and turbulence measurements in the near field of a wingtip vortex. AIAA J., 35, 1561-1567.

Cohn, R. K. and Koochesfahani, M. M. (2000). The accuracy of remapping irregularly spaced velocity data onto a regular grid and the computation of vorticity. Exp. Fluids, Vol. 29, Supplement 1, S061-S069.

Corsiglia, V. R., Schwind, R. G., and Chigier, N. A. (1973). Rapid scanning, threedimensional hotwire anemometer surveys of wing-tip vortices. J. Aircraft, 10, 752-757.

Devenport, W. J., Rife, M. C., Liapis, S. I., and Follin, G. J. (1996). The structure and development of a wing-tip vortex. J. Fluid Mech., 312, 67-106. 
Gerontakos, P and Lee, T. (2006). Near-field tip vortex behind a swept wing model. Exp. Fluids, Vol. 4, No.1, 141-155.

Heyes, A. L., Jones, R. F., and Smith, D. A. R. (2004). Wandering of wing-tip vortices. In Proc. 12th Intl Symp. On the Applications of Laser Techniques to Fluid Mechanics, Lisborn, Portugal.

Hinsch, K. D. (1995). Three-dimensional particle velocimetry. Meas. Sci. Technol., 6, $742-753$.

Hu, H., Saga, T., Kobayashi, T., and Taniguchi, N. (2001). A Study on a Lobed Jet Mixing Flow by Using Stereoscopic PIV Technique. Phy. Fluid, Vol. 13, No.11, 34253441.

Jaquin, L., Fabre, D., Geffroy, P., and Coustols, E. (2001). The properties of a transport aircraft wake in the extended near field: An experimental study. AIAA conference proc., 2001-1038.

Lawson, N. J. and Wu, J. (1997a). Three-dimensional particle image velocimetry: error analysis of stereoscopic techniques. Meas. Sci. Technol., 8, 894-900.

Lawson, N. J. and Wu, J. (1997b). Three-dimensional particle image velocimetry: experimental error analysis of a digital angular stereoscopic system. Meas. Sci. Technol., 8, 1455-1464.

Prasad, A. K. (2000). Stereoscopic particle image velocimetry Exp. Fluids, 29, 103-116.

Prasad A. K., and Adrian R. J. (1993). Stereoscopic Particle Image Velocimetry Applied to Fluid Flows. velocimetry in liquid flows. Exp. Flows, 15, 49-60. 
Prasad, A. K., Adrian, R. J., Landreth, C. C., and Offutt, P. W. (1992). Effect of resolution on the speed and accuracy of particle image velocimetry interrogation. Exp. Fluids, 13, 105-116.

Prasad, A. K. and Jensen K. (1995). Scheimpflug stereocamera for particle image velocimetry in liquid flows. Appl. Opt., 34, 7092-7099.

Ramaprian, B. R., and Zheng, Y.(1997). Measurements in rollup region of the tip vortex from a rectangular wing. velocimetry in liquid flows. AIAA J., 35, 1837-1843.

Rokhsaz, K., Foster, S. R. Miller, L. S. (2000). Exploratory study of aircraft wake vortex filaments in a water tunnel. J. Aircraft, 37, 1022-1027.

Scheimpflug, T. (1904). Improved Method and Apparatus for the Systematic Alteration or Distortion of Plane Pictures and Images by Means of Lenses and Mirrors for Photography and for other purposes. GB Patent No. 1196. Filed 16 January 1904, and issued 12 May 1904.

Soloff, S. M., Adrian, R. J., and Liu, Z-c. (1997). Distorsion compensation for generalized stereoscopic particle image velocimetry. Meas. Sci. Technol., 8, 1441-1454.

Zang, W. and Prasad, A. K. (1997). Performance evaluation of a scheimpflug stereocamera for particle image velocimetry. Appl. Opt., Vol. 36, No. 33, 8738-8744. 